\title{
Quantum stochastic operator cocycles via associated semigroups
}

\author{
BY J. MARTIN LINDSAY \\ Department of Mathematics and Statistics, Lancaster University, Lancaster LA1 4YF. \\ e-mail: j.m.lindsay@lancaster.ac.uk \\ AND STEPHEN J. WILLS \\ School of Mathematical Sciences, University College Cork, Cork, Ireland. \\ e-mail:s.wills@ucc.ie
}

(Received 2 December 2005; revised 4 July 2006)

\begin{abstract}
A recent characterisation of Fock-adapted contraction operator stochastic cocycles on a Hilbert space, in terms of their associated semigroups, yields a general principle for the construction of such cocycles by approximation of their stochastic generators. This leads to new existence results for quantum stochastic differential equations. We also give necessary and sufficient conditions for a cocycle to satisfy such an equation.
\end{abstract}

\section{Introduction}

In this paper we study the functional equation

$$
V_{0}=I, \quad V_{r+t}=V_{r} \sigma_{r}\left(V_{t}\right) \text { for all } r, t \geqslant 0
$$

for a family of contractions on $\mathfrak{h} \otimes \mathcal{F}$ adapted to the Fock operator filtration. Here $\mathcal{F}$ is the symmetric Fock space over $L^{2}\left(\mathbb{R}_{+} ; \mathfrak{k}\right), \mathfrak{h}$ and $\mathrm{k}$ are fixed but arbitrary Hilbert spaces and $\left(\sigma_{t}\right)_{t \geqslant 0}$ is the endomorphism semigroup of shifts, ampliated to $B(\mathfrak{h} \otimes \mathcal{F})$. We call such a family a left contraction cocycle on $\mathfrak{h}$ with noise dimension space $\mathrm{k}$.

Contraction cocycles may be constructed by solving quantum stochastic differential equations of Hudson-Parthasarathy type. By means of a recent characterisation of such cocycles, in terms of an associated family of semigroups (Theorem 1.6), we provide a new method of constructing cocycles which in turn leads to new existence results for QSDEs. When the driving noise is infinite dimensional the coefficient of a QSDE is naturally given as a sesquilinear operator-valued map or, in terms of a coordinate system for the noise dimension space k, as an infinite matrix $\left[F_{\beta}^{\alpha}\right]$. We show that if a process satisfying such a form $Q S D E$ is contractive and strongly measurable then the coefficient is necessarily given by an $o p$ erator, equivalently the matrix must be semiregular. We also give necessary and sufficient conditions, of weak differentiability type, for a strongly continuous left contraction cocycle to satisfy a QSDE.

This paper builds on work of Accardi, Fagnola, Journé, Mohari and the authors ([Fa 2 , Jou, Moh, AJL, FaW]), extending known results for Markov-regular cocycles and QSDEs with bounded coefficients ([HuP, HuL, $\left.\mathbf{L W}, \mathbf{L W}_{\mathbf{2}}\right]$; see $[\mathbf{M e y}, \mathbf{L}]$ and references therein). Our development of the theory is coordinate-free, moreover a technical feature of the work is that 
no separability assumptions are imposed on either the initial space $\mathfrak{h}$ or the noise dimension space k. This freedom is useful for certain applications such as the stochastic dilation of quantum dynamical semigroups $\left(\left[\mathbf{G S}_{\mathbf{1}}\right]\right)$. A different approach to the characterisation and construction of cocycles through semigroup methods has been outlined by Liebscher ([Lie]).

\section{General notations}

The algebraic tensor product is denoted $\otimes$, with $\otimes$ reserved for the tensor product of Hilbert spaces and their operators. For a vector $\xi$ in a Hilbert space K, operators $E_{\xi}: \mathrm{H} \rightarrow$ $\mathrm{H} \otimes \mathrm{K}$ and $E^{\xi}: \mathrm{H} \otimes \mathrm{K} \rightarrow \mathrm{H}$ are defined by

$$
E_{\xi} u=u \otimes \xi \quad \text { and } \quad E^{\xi}=\left(E_{\xi}\right)^{*}
$$

with context indicating the Hilbert space $\mathrm{H}$, and moreover the elementary tensor $u \otimes \xi$ is usually abbreviated to $u \xi$. Note that $\xi \mapsto E_{\xi}$ is an isometry. For Hilbert spaces $H$ and $H^{\prime}$ and a dense subspace $\mathcal{D}$ of $\mathrm{H}, \mathcal{O}\left(\mathcal{D} ; \mathrm{H}^{\prime}\right)$ denotes the linear space of operators $\mathrm{H} \rightarrow \mathrm{H}^{\prime}$ with domain $\mathcal{D} ; \mathcal{O}(\mathcal{D})$ abbreviates $\mathcal{O}(\mathcal{D} ; \mathrm{H})$. For $f \in L^{2}\left(\mathbb{R}_{+} ; \mathrm{k}\right)$ and $I \subset \mathbb{R}_{+}, f_{I}$ denotes the function that agrees with $f$ on $I$ and is zero elsewhere, and $c_{I}$ denotes the function equal to $c$ on $I$ and zero elsewhere, when $c$ is a vector in $\mathrm{k}$.

\section{Fock space}

We use normalised exponential vectors in $\mathcal{F}=\Gamma\left(L^{2}\left(\mathbb{R}_{+} ; \mathrm{k}\right)\right)$, the symmetric Fock space over the Hilbert space $L^{2}\left(\mathbb{R}_{+} ; \mathrm{k}\right)$. These are defined by $\varpi(f):=\|\varepsilon(f)\|^{-1} \varepsilon(f)$ where $\varepsilon(f)=\left(1, f,(2 !)^{-1 / 2} f^{\otimes 2}, \ldots\right)$ for $f \in L^{2}\left(\mathbb{R}_{+} ; \mathrm{k}\right)$. The function

$$
\chi: \mathrm{k} \times \mathrm{k} \rightarrow \mathbb{C}, \quad(c, d) \mapsto \frac{1}{2}\left(\|c\|^{2}+\|d\|^{2}\right)-\langle c, d\rangle,
$$

which governs their inner product: $\langle\varpi(f), \varpi(g)\rangle=\exp \left(-\int \chi(f(s), g(s)) d s\right)$, also plays a role. The subspace $\mathcal{E}(S):=\operatorname{Lin}\{\varepsilon(f): f \in S\}$ is dense in $\mathcal{F}$ for various useful subsets of $L^{2}\left(\mathbb{R}_{+} ; \mathrm{k}\right)$, for example

$$
\mathbb{S}_{\mathrm{T}}:=\{f \in \mathbb{S}: f \text { is } \mathbf{T} \text {-valued }\}
$$

where $\mathbb{S}:=\operatorname{Lin}\left\{c_{[0, t[}: c \in \mathrm{k}, t>0\right\}$, and $\mathrm{T}$ is a total subset of $\mathrm{k}$ containing 0 ; we write $\mathcal{E}_{\mathrm{T}}$ for $\mathcal{E}\left(\mathbb{S}_{\mathrm{T}}\right)$. Examples of such sets $\mathrm{T}$ include (not necessarily normalised) orthogonal bases, augmented by 0 .

We shall need a refinement of the basic estimate

$$
\|\varpi(f)-\varpi(g)\| \leqslant\|\varepsilon(f)-\varepsilon(g)\| \leqslant\|f-g\| e^{\frac{1}{2}(\|f\|+\|g\|)^{2}}
$$

obtained by viewing $L^{2}\left(\mathbb{R}_{+} ; \mathrm{k}\right)$ as a subspace of $\mathcal{F}$, namely

$$
\|\varepsilon(f)-\varepsilon(g)-(f-g)\| \leqslant\|f-g\|(\|f\|+\|g\|) e^{\frac{1}{2}(\|f\|+\|g\|)^{2}} .
$$

Letting $\mathcal{F}_{I}$ denote the symmetric Fock space over $L^{2}(I ; \mathrm{k})$, for a subinterval $I$ of $\mathbb{R}_{+}$, the tensor factorisation $\mathcal{F} \cong \mathcal{F}_{[0, t[} \otimes \mathcal{F}_{[t, \infty[}$ is given by continuous linear extension of the correspondence

$$
\varpi(f) \longleftrightarrow \varpi\left(\left.f\right|_{[0, t[}\right) \varpi\left(\left.f\right|_{[t, \infty[}\right)
$$

\section{Operator processes}

A family of operators $\left(X_{t}\right)_{t \geqslant 0}$ in $B(\mathfrak{h} \otimes \mathcal{F})$ is adapted if

$$
X_{t} \in B\left(\mathfrak{h} \otimes \mathcal{F}_{[0, t]}\right) \otimes I_{\mathcal{F}_{[t, \infty[}} \quad \text { for all } t,
$$


that is, if each $X_{t}$ only acts nontrivially before time $t$, and is called an (operator) process if furthermore it is weakly measurable (i.e. if $t \mapsto\left\langle\xi, X_{t} \zeta\right\rangle$ is measurable for all $\xi, \zeta \in \mathfrak{h} \otimes \mathcal{F}$ ). Here we are concerned with contraction operator-valued processes, which we refer to simply as contraction processes on $\mathfrak{h}$.

The right shift $s_{t}$ and time reversal map $r_{t}$ on $L^{2}\left(\mathbb{R}_{+} ; \mathrm{k}\right)$ are

$$
\left(s_{t} f\right)(u)=\left\{\begin{array}{ll}
0 & \text { if } u<t, \\
f(u-t) & \text { if } u \geqslant t,
\end{array} \quad \text { and } \quad\left(r_{t} f\right)(u)= \begin{cases}f(t-u) & \text { if } u \leqslant t \\
f(u) & \text { if } u>t\end{cases}\right.
$$

Their second quantisations ampliated to $\mathfrak{h} \otimes \mathcal{F}$ are the isometry $S_{t}$ and self-adjoint unitary $R_{t}$ respectively, given by

$$
S_{t} u \varepsilon(f)=u \varepsilon\left(s_{t} f\right), \quad R_{t} u \varepsilon(f)=u \varepsilon\left(r_{t} f\right) .
$$

Thinking of $s_{t}$ as a unitary $L^{2}\left(\mathbb{R}_{+} ; \mathrm{k}\right) \rightarrow L^{2}([t, \infty[; \mathrm{k})$ we get the Hilbert space isomorphism $\mathcal{F} \cong \mathcal{F}_{[t, \infty[}$, and the algebra isomorphism $B(\mathfrak{h} \otimes \mathcal{F}) \cong B\left(\mathfrak{h} \otimes \mathcal{F}_{[t, \infty[}\right)$. The latter algebra is viewed as a subalgebra of the former:

$$
B(\mathfrak{h} \otimes \mathcal{F}) \cong B(\mathfrak{h}) \otimes I_{\mathcal{F}_{[0, t]}} \otimes B\left(\mathcal{F}_{[t, \infty[}\right) \subset B(\mathfrak{h} \otimes \mathcal{F})
$$

Then, for $Y \in B(\mathfrak{h} \otimes \mathcal{F}), \sigma_{t}(Y) \in B(\mathfrak{h} \otimes \mathcal{F})$ denotes the result of carrying out these identifications; more concretely it is determined by the identity

$$
\left\langle u \varepsilon(f), \sigma_{t}(Y) v \varepsilon(g)\right\rangle=\left\langle u \varepsilon\left(s_{t}^{*} f\right), Y v \varepsilon\left(s_{t}^{*} g\right)\right\rangle\left\langle\varepsilon\left(f_{[0, t]}\right), \varepsilon\left(g_{[0, t]}\right)\right\rangle .
$$

The family $\left(\sigma_{t}\right)_{t \geqslant 0}$ is a pointwise weakly continuous semigroup of normal endomorphisms of $B(\mathfrak{h} \otimes \mathcal{F})$.

\section{Perturbation}

We end this introduction by quoting a dissipative generalisation of the Kato-Rellich Theorem whose symmetric form is well-suited to our purposes. Recall that a $C_{0}$-semigroup is contractive if and only if its generator is dissipative.

THEOREM 0.1. Let $A$ and $B$ be densely defined dissipative operators on a Banach space with the same domain $\mathcal{D}$, and suppose that there are constants $\lambda, \mu \geqslant 0$ with $\lambda<1$ such that their difference $D=A-B$ satisfies

$$
\|D v\| \leqslant \lambda(\|A v\|+\|B v\|)+\mu\|v\|, \quad v \in \mathcal{D} .
$$

Then Dom $\bar{A}=\operatorname{Dom} \bar{B}$, moreover $\bar{A}$ is a $C_{0}$-semigroup generator if and only if $\bar{B}$ is.

This result is due to Gustafson (see [ReS, theorem X.50]). Note that if $(0 \cdot 5)$ holds then $D$ is $A$-bounded with relative bound at most $2 \lambda /(1-\lambda)$.

\section{Cocycles and semigroups}

Left contraction cocycles on $\mathfrak{h}$ have been defined in the introduction. An adapted family of contractions $U=\left(U_{t}\right)_{t \geqslant 0}$ on $\mathfrak{h} \otimes \mathcal{F}$, satisfying $U_{0}=I$ and $U_{r+t}=\sigma_{r}\left(U_{t}\right) U_{r}$ for $r, t \geqslant 0$, is called a right contraction cocycle. Thus $U$ is a right contraction cocycle if and only if $U^{*}:=\left(U_{t}^{*}\right)_{t \geqslant 0}$ is a left contraction cocycle.

The semigroup decomposition

For a contraction process $V=\left(V_{t}\right)_{t \geqslant 0}$ on $\mathfrak{h}$, define the following operators on $\mathfrak{h}$ :

$$
{ }^{V} Q_{t}^{c, d}=E^{\varpi\left(c_{[0, t]}\right)} V_{t} E_{\varpi\left(d_{[0, t]}\right)}, \quad c, d \in \mathrm{k}, t \geqslant 0 .
$$


These 'sliced' operators allow one to determine whether or not a process $V$ is a left contraction cocycle. Note that they are all contractions.

Proposition 1.1 ([LW $\left.\left.\mathbf{L W}_{2}\right]\right)$. Let $V$ be a contraction process on $\mathfrak{h}$, and let $\mathrm{T}$ and $\mathrm{T}^{\dagger}$ be any total subsets of $\mathrm{k}$ containing 0 . Then the following are equivalent:

(i) $V$ is a left contraction cocycle;

(ii) for each choice of $c \in \mathrm{T}^{\dagger}$ and $d \in \mathrm{T},\left(Q_{t}^{c, d}:={ }^{V} Q_{t}^{c, d}\right)_{t \geqslant 0}$ is a contraction semigroup on $\mathfrak{h}$, and for all $f \in \mathbb{S}_{T^{\dagger}}$ and $g \in \mathbb{S}_{\top}$

$$
E^{\varpi\left(f_{[0, t]}\right)} V_{t} E_{\varpi\left(g_{[0, t]}\right)}=Q_{t_{1}-t_{0}}^{f\left(t_{0}\right), g\left(t_{0}\right)} \cdots Q_{t-t_{n}}^{f\left(t_{n}\right), g\left(t_{n}\right)}
$$

where $\left\{0=t_{0} \leqslant t_{1} \leqslant \cdots \leqslant t_{n} \leqslant t\right\}$ contains the discontinuities of $f_{[0, t[}$ and $g_{[0, t[}$, and right-continuous versions are used for the evaluations.

We refer to $\left\{{ }^{V} Q^{c, d}: c, d \in \mathrm{k}\right\}$ as the cocycle's associated semigroups.

Remark. The same holds for right contraction cocycles except that the product in $(1 \cdot 1)$ is in the reverse order. It follows that $\left(U_{t}\right)_{t \geqslant 0}$ is a right contraction cocycle if and only if $\left(R_{t} U_{t} R_{t}\right)_{t \geqslant 0}$ defines a left contraction cocycle, where the operators $R_{t}$ are defined in (0.4).

For a left contraction cocycle $V$, we refer to the left contraction cocycle defined by $\left(R_{t} V_{t}^{*} R_{t}\right)_{t \geqslant 0}$ as the (Journé) dual of $V$ ([Jou]), and denote it $\widetilde{V}$. The associated semigroups of the dual cocycle are related to those of $V$ as follows:

$$
\widetilde{Q}_{t}^{c, d}=\left(Q_{t}^{d, c}\right)^{*} .
$$

\section{Continuity}

The above proposition makes no continuity demands on the time variable of $V$ - indeed it does not even require the weak measurability condition imposed on processes. However the decomposition of a cocycle into its associated semigroups does provide a useful handle on the continuity of a cocycle.

Lemma 1.2. Let $V$ be a left contraction cocycle on $\mathfrak{h}$ and let $\left\{Q^{c, d}: c, d \in \mathrm{k}\right\}$ be its associated semigroups. Then, the following are equivalent:

(i) $V$ is strongly continuous;

(ii) $V$ is weakly continuous at 0 ;

(iii) for all $c, d \in \mathrm{k}, Q^{c, d}$ is strongly continuous;

(iv) for some $a, b \in \mathrm{k}, Q^{a, b}$ is weakly continuous at 0 ;

Proof. Let $c, d \in \mathrm{k}$ and suppose that $V$ is weakly continuous at 0 . By adaptedness

$$
\left\langle u, Q_{t}^{c, d} v\right\rangle=\left\langle\varpi\left(c_{[t, T[}\right), \varpi\left(d_{[t, T[}\right)\right\rangle^{-1}\left\langle u \varpi\left(c_{[0, T[}\right), V_{t} v \varpi\left(d_{[0, T[}\right)\right\rangle,
$$

for $0 \leqslant t \leqslant T$ and $u, v \in \mathfrak{h}$, and so the contraction semigroup $Q^{c, d}$ is weakly continuous at 0 and thus also strongly continuous, by standard semigroup theory ([Dav, proposition 1.23]). Thus (ii) implies (iii). Suppose now that $Q^{a, b}$ is weakly continuous at 0 (and thus strongly continuous) for some $a, b \in \mathrm{k}$, and let $t \geqslant r$ and $\xi=v \varpi(f)$ for $v \in \mathfrak{h}$ and $f \in L^{2}\left(\mathbb{R}_{+} ; \mathrm{k}\right)$. Then by the cocycle relation, and contractivity and adaptedness of $V$,

$$
\begin{aligned}
\left\|V_{t} \xi-V_{r} \xi\right\|^{2} & \leqslant\left\|\sigma_{r}\left(V_{t-r}\right) \xi-\xi\right\|^{2} \\
& \leqslant 2 \operatorname{Re}\left\langle\xi,\left(I-\sigma_{r}\left(V_{t-r}\right)\right) \xi\right\rangle \\
& =2 \operatorname{Re}\left\langle S_{r}^{*} v \varpi\left(f_{[r, t]}\right),\left(I-V_{t-r}\right) S_{r}^{*} v \varpi\left(f_{[r, t]}\right)\right\rangle .
\end{aligned}
$$


For any $e \in \mathrm{k}$ let $\varphi_{r, t}^{e}=\varpi\left(f_{[r, t]}\right)-\varpi\left(e_{[r, t[}\right)$, so $S_{r}^{*} v \varpi\left(f_{[r, t[}\right)=S_{r}^{*} v \varphi_{r, t}^{e}+v \varpi\left(e_{[0, t-r[}\right)$, and thus the right-hand side of the above is no larger than

$$
4\|v\|^{2}\left\{\left\|\varphi_{r, t}^{a}\right\|\left\|\varphi_{r, t}^{b}\right\|+\left\|\varphi_{r, t}^{a}\right\|+\left\|\varphi_{r, t}^{b}\right\|\right\}+2\left|\left\langle v \varpi\left(a_{[0, t-r[}\right),\left(I-V_{t-r}\right) v \varpi\left(b_{[0, t-r[}\right)\right)\right| .
$$

The first term converges to 0 as $t-r \rightarrow 0$, and the second term equals

$$
2\left|\left\langle v,\left(I-Q_{t-r}^{a, b}\right) v\right\rangle+\|v\|^{2}\{\exp (r-t) \chi(a, b)-1\}\right|,
$$

hence $\left\|\left(V_{t}-V_{r}\right) \xi\right\| \rightarrow 0$ as $t-r \rightarrow 0$ by the assumption on $Q^{a, b}$. Therefore, since the collection of such vectors $\xi$ is total, the uniform boundedness of $V$ implies that it is strongly continuous. Thus (iv) implies (i). Since the implications (i) $\Rightarrow$ (ii) and (iii) $\Rightarrow$ (iv) are trivial the proof is complete.

Thus, as for semigroups, strong continuity for a left contraction cocycle is equivalent to weak continuity at 0 , and also to any of its associated semigroups - in particular its Markov semigroup $Q^{0,0}$ - being a $C_{0}$-semigroup.

Remark. By the strong continuity of $t \mapsto R_{t}$, Lemma 1.2 is equally true for right contraction cocycles.

Suppose that $V$ is a strongly continuous left contraction cocycle on $\mathfrak{h}$. Then each ${ }^{V} Q^{c, d}$ is a $C_{0}$-contraction semigroup by Lemma 1.2 and so has a generator $G_{c, d}^{V}$. For immediate purposes it is convenient to also work with the $C_{0}$-semigroups defined by

$$
{ }^{V} P_{t}^{c, d}:=E^{\varepsilon\left(c_{[0, t[}\right)} V_{t} E_{\varepsilon\left(d_{[0, t]}\right)}=e^{t\left(\|c\|^{2}+\|d\|^{2}\right) / 2}{ }^{V} Q_{t}^{c, d}, \quad c, d \in \mathrm{k},
$$

and their generators, which we denote $H_{c, d}^{V}$. The generators are related by

$$
H_{c, d}^{V}-\langle c, d\rangle=G_{c, d}^{V}+\chi(c, d),
$$

with equality of domains, where $\chi$ is the function defined in $(0 \cdot 1)$.

Note also that, from $(1 \cdot 2)$,

$$
H_{c, d}^{\widetilde{V}}=\left(H_{d, c}^{V}\right)^{*} \text { and } G_{c, d}^{\widetilde{V}}=\left(G_{d, c}^{V}\right)^{*}
$$

Operators associated with a cocycle

In this section the generators of semigroups associated with a strongly continuous left contraction cocycle are compared. This will lead to natural sufficient conditions on their domains for such a cocycle to be governed by a QSDE. First note two consequences of the estimates $(0 \cdot 2)$ and $(0 \cdot 3)$. For locally bounded functions $f$ and $g$ in $L^{2}\left(\mathbb{R}_{+} ; \mathrm{k}\right)$,

$$
\begin{aligned}
& \left\|\varepsilon\left(f_{[s, t[}\right)-\varepsilon\left(g_{[s, t[}\right)\right\|=O(\sqrt{t-s}) \text { and } \\
& \left\|\varepsilon\left(f_{[s, t[}\right)-\varepsilon\left(g_{[s, t[}\right)-(f-g)_{[s, t[}\right\|=O(t-s),
\end{aligned}
$$

as $(t-s) \rightarrow 0$ with $[s, t$ [ in some finite interval. In particular, for $a, c \in \mathrm{k}$,

$$
\begin{aligned}
& \left\|\varepsilon\left(c_{[s, t[}\right)-\varepsilon\left(a_{[s, t[}\right)\right\|=O(\sqrt{t-s}) \text { and } \\
& \left\|\varepsilon\left(c_{[s, t]}\right)-\varepsilon\left(a_{[s, t]}\right)-(c-a)_{[s, t[}\right\|=O(t-s) .
\end{aligned}
$$

The former refines to

$$
(t-s)^{-1 / 2}\left\|\varepsilon\left(c_{[s, t[}\right)-\varepsilon\left(a_{[s, t]}\right)\right\| \leqslant\|c-a\|+O(t-s) .
$$


Viewing $\mathfrak{h} \otimes \mathrm{k} \otimes L^{2}\left(\mathbb{R}_{+}\right)=\mathfrak{h} \otimes L^{2}\left(\mathbb{R}_{+} ; \mathrm{k}\right)$ as a subspace of $\mathfrak{h} \otimes \mathcal{F}$, define two families of operators associated with a cocycle $V$ :

$$
\begin{aligned}
& T_{d}^{V}(t):=t^{-1} E^{1_{[0, t]}} V_{t} E_{\varepsilon\left(d_{[0, t]}\right]}, \text { and } \\
& C^{V}(t):=t^{-1} E^{1_{[0, t]}} V_{t} E_{1_{[0, t}},
\end{aligned}
$$

for $d \in \mathrm{k}$ and $t>0$. Thus $T_{d}^{V}(t) \in B(\mathfrak{h} ; \mathfrak{h} \otimes \mathrm{k})$, and $C^{V}(t) \in B(\mathfrak{h} \otimes \mathbf{k})$ is a contraction. Since its associated semigroups satisfy

$$
\begin{aligned}
\left\langle u,\left({ }^{V} P_{t}^{c, d}-{ }^{V} P_{t}^{a, d}\right) v\right\rangle-t & \left\langle u(c-a), T_{d}^{V}(t) v\right\rangle \\
& =\left\langle u\left(\varepsilon\left(c_{[0, t]}\right)-\varepsilon\left(a_{[0, t]}\right)-(c-a)_{[0, t]}\right), V_{t} v \varepsilon\left(d_{[0, t]}\right)-v \varepsilon(0)\right\rangle
\end{aligned}
$$

and $V_{t} v \varepsilon\left(d_{[0, t[}\right) \rightarrow v \varepsilon(0)$ as $t \rightarrow 0^{+}$, the estimate (1.5) implies that

$$
t\left\langle u(c-a), T_{d}^{V}(t) v\right\rangle=\left\langle u,\left({ }^{V} P_{t}^{c, d}-{ }^{V} P_{t}^{a, d}\right) v\right\rangle+o(t)
$$

as $t \rightarrow 0$; similarly

$$
t\left\langle u(c-a), C^{V}(t) v(d-b)\right\rangle=\left\langle u,\left({ }^{V} P_{t}^{c, d}-{ }^{V} P_{t}^{c, b}-{ }^{V} P_{t}^{a, d}+{ }^{V} P_{t}^{a, b}\right) v\right\rangle+O\left(t^{3 / 2}\right) .
$$

Now define operators $T_{d}^{V}$ and $C^{V}$ by

$$
T_{d}^{V} v=\underset{t \rightarrow 0^{+}}{\mathrm{w}-\lim _{d}} T_{d}^{V}(t) v \text { and } C^{V} \xi=\underset{t \rightarrow 0^{+}}{\mathrm{w}-\lim ^{V}} C^{V}(t) \xi
$$

with domains equal to the subspaces on which weak convergence holds. Thus Dom $C^{V}$ is a closed subspace of $\mathfrak{h} \otimes \mathrm{k}$ on which $C^{V}$ is a contraction. We shall see that each $T_{d}^{V}$ is densely defined and obtain sufficient conditions for $C^{V}$ to be defined on all of $\mathfrak{h} \otimes \mathrm{k}$.

For a subset $S$ of k and element $d$ of k, define

$$
\mathfrak{D}^{V, d}:=\operatorname{Dom} G_{d, d}^{V}, \text { and } \mathfrak{D}^{V, S}:=\bigcap_{c \in S} \mathfrak{D}^{V, c} .
$$

Note that Dom $H_{c, d}^{V}=\operatorname{Dom} G_{c, d}^{V}$ for all $c, d \in \mathrm{k}$.

In the next result we shall spare the reader a panoply of symbols by dropping the $V$ and $\widetilde{V}$ superscripts, writing $\widetilde{T}_{c}$ for $T_{c}^{\widetilde{V}}$ and so forth.

PROPOSITION 1-3. Let $V$ be a strongly continuous left contraction cocycle on $\mathfrak{h}$ with noise dimension space $\mathrm{k}$, let $c, d \in \mathrm{k}$.

(a) For each $v \in \mathfrak{D}^{d}$ and $f \in\left(L^{2} \cap L_{\mathrm{loc}}^{\infty}\right)\left(\mathbb{R}_{+} ; \mathrm{k}\right)$, the map $t \mapsto V_{t} v \varepsilon(f)$ is (locally) Hölder continuous with exponent $1 / 2$.

(b) For each $a \in \mathrm{k}$, Dom $H_{a, d}=\mathfrak{D}^{d}$. Moreover, for any dense subspace $\mathfrak{D}$ of $\mathfrak{D}^{d}$, $\mathfrak{D}$ is a core for $H_{c, d}$ if and only if it is a core for $H_{d, d}$.

(c) For each e $\in \mathrm{k}$,

$$
E^{e} T_{d} \supset H_{c+e, d}-H_{c, d} .
$$

In particular, the map $c \mapsto H_{c, d}$ is complex-conjugate affine linear.

(d) For all $b, e \in \mathrm{k}$,

$$
H_{c, d}-H_{c, b} \subset\left(\widetilde{T}_{c}\right)^{*} E_{d-b} \text { and } H_{c, d}+\left(\widetilde{T}_{c}\right)^{*} E_{e} \subset H_{c, d+e},
$$

and the maps $d \mapsto H_{c, d}$ and $d \mapsto T_{d}$ are complex affine linear in the sense that, for all $b \in \mathrm{k}$ and $z \in \mathbb{C}$, if $e=(1-z) b+z d$ then

$$
H_{c, e} \supset(1-z) H_{c, b}+z H_{c, d} \text { and } T_{e} \supset(1-z) T_{b}+z T_{d} .
$$


(e) For $b, e \in \mathrm{k}$

$$
T_{d}-T_{b} \subset C E_{d-b} \text { and } T_{d}+C E_{e} \subset T_{d+e},
$$

in particular the operator $\left(T_{d}-T_{b}\right)$ is bounded on its domain. If Dom $C E_{d}=\mathfrak{h}$ then $\left(C E_{d}\right)^{*} \supset E^{d} \widetilde{C}$. Also Dom $C=\mathfrak{h} \otimes \mathrm{k}$ if and only if Dom $\widetilde{C}=\mathfrak{h} \otimes \mathrm{k}$, in which case $\widetilde{C}=C^{*}$ and Dom $T_{d}$ is independent of $d$.

(f) For each $b \in \mathrm{k}, T_{b}$ is $H_{c, d}$-bounded, with relative bound 0 , on $\mathfrak{D}^{d} \cap \operatorname{Dom} T_{b} \supset \mathfrak{D}^{\{b, d\}}$, in the notation (1.9).

Proof. First note that for $v \in \mathfrak{D}^{d}$, and $\lambda, t>0$,

$$
\begin{aligned}
t^{-1}\left\|\left(V_{t}-I\right) v \varpi\left(d_{[0, t]}\right)\right\|^{2} & \leqslant 2 t^{-1} \operatorname{Re}\left\langle v,\left(I-Q_{t}^{d, d}\right) v\right\rangle \\
& =-2 t^{-1} \operatorname{Re} \int_{0}^{t}\left\langle v, Q_{s}^{d, d} G_{d, d} v\right\rangle d s \\
& \leqslant 2\|v\|\left\|G_{d, d} v\right\| \\
& \leqslant 2\left(\|v\|\left\|H_{d, d} v\right\|+\|d\|^{2}\|v\|^{2}\right) \\
& \leqslant\left(\lambda\left\|H_{d, d} v\right\|+\left(\lambda^{-1}+\sqrt{2}\|d\|\right)\|v\|\right)^{2},
\end{aligned}
$$

SO

$$
t^{-1 / 2}\left\|\left(V_{t}-I\right) v \varepsilon\left(d_{[0, t[}\right)\right\| \leqslant \lambda\left\|H_{d, d} v\right\|+\left(\lambda^{-1}+\sqrt{2}\|d\|\right)\|v\|+O(t)
$$

as $t \rightarrow 0$. In particular, since $E^{1_{[0, t]}} E_{\varepsilon(0)}=0$, using (1.6)

$$
\begin{aligned}
\left\|T_{d}(t) v\right\| & \leqslant t^{-1 / 2}\left\|V_{t} v \varepsilon\left(d_{[0, t[}\right)-v \varepsilon(0)\right\| \\
& \leqslant \lambda\left\|H_{d, d} v\right\|+\mu(\lambda)\|v\|+O(t)
\end{aligned}
$$

where $\mu(\lambda)=\lambda^{-1}+(1+\sqrt{2})\|d\|$. From $(1 \cdot 7)$ therefore

$$
\begin{aligned}
\left\|\left(P_{t}^{c, d}-P_{t}^{a, d}\right) v\right\| & \leqslant t\|c-a\|\left\|T_{d}(t) v\right\|+o(t) \\
& \leqslant t\|c-a\|\left(\lambda\left\|H_{d, d} v\right\|+\mu(\lambda)\|v\|\right)+o(t) .
\end{aligned}
$$

(a) Let $v \in \mathfrak{D}^{d}, f \in\left(L^{2} \cap L_{\mathrm{loc}}^{\infty}\right)\left(\mathbb{R}_{+} ; \mathrm{k}\right)$ and $T \geqslant t \geqslant s \geqslant 0$. Then, from the estimate (1·3) the function $f_{s, t}:=f+(d-f)_{[s, t[}$ satisfies

$$
\left\|\varepsilon(f)-\varepsilon\left(f_{s, t}\right)\right\|=O(\sqrt{t-s}),
$$

as $(t-s) \rightarrow 0$. Using the cocycle and adaptedness properties of $V$,

$$
\begin{aligned}
\left\|\left(V_{t}-V_{s}\right) v \varepsilon\left(f_{s, t}\right)\right\| & \leqslant\left\|\sigma_{s}\left(V_{t-s}-I\right) v \varepsilon\left(f_{s, t}\right)\right\| \\
& =\left\|\varepsilon\left(f_{\left[s, t\left[^{c}\right.\right.}\right)\right\|\left\|\sigma_{s}\left(V_{t-s}-I\right) v \varepsilon\left(d_{[s, t[}\right)\right\| \\
& \leqslant\|\varepsilon(f)\|\left\|\left(V_{t-s}-I\right) v \varepsilon\left(d_{[0, t-s[}\right)\right\| .
\end{aligned}
$$

Therefore, by (1·13), if follows that $\left\|\left(V_{t}-V_{s}\right) v \varepsilon(f)\right\|=O(\sqrt{t-s})$, and (a) follows.

(b) By $(1 \cdot 15)$

$$
\limsup _{t \rightarrow 0^{+}} t^{-1}\left\|\left(P_{t}^{c, d}-P_{t}^{d, d}\right) v\right\|<\infty \text { for } v \in \mathfrak{D}^{d} .
$$

By standard semigroup theory ([Dav, corollary 1.39]) it follows that Dom $H_{c, d} \supset \mathfrak{D}^{d}$, and (1.15) gives

$$
\left\|\left(H_{c, d}-H_{d, d}\right) v\right\| \leqslant\|c-d\|\left(\lambda\left\|H_{d, d} v\right\|+\mu(\lambda)\|v\|\right), \quad v \in \mathfrak{D}^{d} .
$$


We may therefore apply Gustafson's Theorem (Theorem 0.1) with $A=\left.H_{c, d}\right|_{\mathfrak{D}^{d}}, B=H_{d, d}$ and an appropriately chosen $\lambda$ to conclude that

$$
\text { Dom } \bar{A}=\operatorname{Dom} \bar{B}=\operatorname{Dom} B=\operatorname{Dom} A
$$

and that $A$ itself generates a $C_{0}$-contraction semigroup. But $C_{0}$-contraction semigroup generators are maximal dissipative ([Dav, theorem 6.4] or [ReS, page 241]) so the inclusion $H_{c, d} \supset A$ is an equality - in other words Dom $H_{c, d}=\mathfrak{D}^{d}$. A further application of Gustafson's Theorem now shows that, for a dense subspace $\mathfrak{D}$ of $\mathfrak{D}^{d}, \mathfrak{D}$ is a core for $H_{c, d}$ if and only if it is a core for $H_{d, d}$.

(c) Let $v \in \mathfrak{D}^{d}$. First note that, by (1.14), $T_{d}(t) v$ is locally bounded in $t$ in a neighbourhood of 0 . Thus, in view of (b) and (1.7), $v \in$ Dom $T_{d}$ and $E^{e} T_{d} v=\left(H_{c+e, d}-H_{c, d}\right) v$ for any $e \in \mathrm{k}$. Thus $(1 \cdot 10)$ holds.

(d) Let $b \in \mathrm{k}, v \in \mathfrak{D}^{\{b, d\}}$ and $u \in \operatorname{Dom} \widetilde{T}_{c}$. Then, for $t>0$,

$$
\left\langle v(d-b), \widetilde{T}_{c}(t) u\right\rangle=t^{-1}\left\langle v,\left(\widetilde{P}_{t}^{d, c}-\widetilde{P}_{t}^{b, c}\right) u\right\rangle+o(1)=t^{-1}\left\langle\left(P_{t}^{c, d}-P_{t}^{c, b}\right) v, u\right\rangle+o(1) .
$$

It follows that $v(d-b) \in \operatorname{Dom}\left(\widetilde{T}_{c}\right)^{*}$ and $\left(\widetilde{T}_{c}\right)^{*} E_{d-b} v=\left(H_{c, d}-H_{c, b}\right) v$. This proves the first inclusion in (1.11); (1.10) applied to $\widetilde{V}$ gives $\widetilde{H}_{d, c}+E^{e} \widetilde{T}_{c}=\widetilde{H}_{d+e, c}$ which yields the second:

$$
H_{c, d+e}=\left(\widetilde{H}_{d, c}+E^{e} \widetilde{T}_{c}\right)^{*} \supset H_{c, d}+\widetilde{T}_{c}^{*} E_{e} .
$$

By (c), setting $e=(1-z) b+z d$,

$$
(1-z) H_{c, b}+z H_{c, d} \subset\left((1-\bar{z}) \tilde{H}_{b, c}+\bar{z} \widetilde{H}_{d, c}\right)^{*}=\left(\tilde{H}_{e, c}\right)^{*}=H_{c, e} .
$$

This gives the first of the inclusions (1.12); the second follows from the observation

$$
\begin{aligned}
\left\|(1-z) T_{b}(t)+z T_{d}(t)-T_{e}(t)\right\| & \leqslant t^{-1 / 2}\left\|(1-z) \varepsilon\left(b_{[0, t]}\right)+z \varepsilon\left(d_{[0, t[}\right)-\varepsilon\left(e_{[0, t[}\right)\right\| \\
& =O\left(t^{1 / 2}\right) .
\end{aligned}
$$

(e) The first two inclusions follow from the observation

$$
\begin{aligned}
\left\|T_{d}(t)-T_{b}(t)-C(t) E_{d-b}\right\| & \leqslant t^{-1 / 2}\left\|\varepsilon\left(d_{[0, t[}\right)-\varepsilon\left(b_{[0, t[}\right)-(d-b)_{[0, t[}\right\| \\
& =O\left(t^{1 / 2}\right),
\end{aligned}
$$

by $(1 \cdot 5)$, and the rest follows from the fact that $\widetilde{C}(t)=C(t)^{*}$ for each $t>0$.

(f) Let $v \in \mathfrak{D}^{d}$ and $\lambda>0$. From $(1 \cdot 15)$

$$
\left\|\left(H_{c, d}-H_{a, d}\right) v\right\| \leqslant\|c-a\|\left(\lambda\left\|H_{d, d} v\right\|+\mu(\lambda)\|v\|\right) .
$$

Taking $a=d$ it follows that

$$
(1-\|c-d\| \lambda)\left\|H_{d, d} v\right\| \leqslant\left\|H_{c, d} v\right\|+\|c-d\| \mu(\lambda)\|v\| .
$$

But from (1.14) it follows that

$$
\left\|T_{d} v\right\| \leqslant \lambda\left\|H_{d, d} v\right\|+\mu(\lambda)\|v\|,
$$

therefore $T_{d}$ is $H_{c, d}$-bounded with relative bound 0 . Since $\left(T_{d}-T_{b}\right)$ is bounded on its domain (f) follows. This completes the proof.

To a strongly continuous left contraction cocycle $V$ on $\mathfrak{h}$, with noise dimension space $\mathrm{k}$, we may therefore associate an operator on $\mathfrak{h} \oplus(\mathfrak{h} \otimes \mathbf{k})$ by

$$
F^{V}:=\left[\begin{array}{cc}
Z^{V} & M^{V} \\
L^{V} & C^{V}-I
\end{array}\right]
$$


where $Z^{V}=H_{0,0}^{V}, L^{V}=\left.T_{0}^{V}\right|_{\mathfrak{D}^{V, 0}}$ and $M^{V}=\left(\left.T_{0}^{\widetilde{V}}\right|_{\mathfrak{D}^{\tilde{V}, 0}}\right)^{*}$. Thus $Z^{V}$ is a $C_{0}$-contraction semigroup generator, $L^{V}$ has the same dense domain as $Z^{V}, M^{V}$ is closed and $C^{V}$ is a contraction operator. If $F^{V}$ is densely defined then

$$
\left(F^{V}\right)^{*} \supset F^{\widetilde{V}}
$$

COROLlary 1.4. For all $c, d \in \mathrm{k}$ and $S \subset \mathrm{k}$

$$
\text { Dom } G_{c, d}^{V}=\mathfrak{D}^{V, d} \text { and } \mathfrak{D}^{V, \text { Aff } S}=\mathfrak{D}^{V, S}
$$

where Aff $S$ denotes the complex affine span of $S$. Moreover,

$$
\operatorname{Dom} F^{V} \supset \mathfrak{D}^{V, 0} \oplus\left(\mathfrak{D}^{V, \text { Aff } S} \underline{\otimes} \mathbf{D}\right),
$$

where $\mathrm{D}=\operatorname{Lin}(S-S)$.

Proof. For convenience we drop the superscripts $V$ and $\widetilde{V}$ as in the proposition. The semigroup generators $G_{c, d}$ and $H_{c, d}$ have the same domains, so the first equality follows from part (b) of the proposition. For the second equality, if $e \in \operatorname{Aff} S$ then $\mathfrak{D}^{e} \supset \mathfrak{D}^{S}$ by the first inclusion in (1-12). But this implies that

$$
\mathfrak{D}^{S} \subset \bigcap_{e \in \text { Aff } S} \mathfrak{D}^{e}=\mathfrak{D}^{\text {Aff } S} \subset \mathfrak{D}^{S} .
$$

For $(1 \cdot 17)$ note that if $b, d \in \mathrm{k}$ then

$$
M E_{d-b}=\left(\left.E^{d-b} \widetilde{T}_{0}\right|_{\mathfrak{D}^{0}}\right)^{*}=\left(\widetilde{H}_{d, 0}-\widetilde{H}_{b, 0}\right)^{*} \supset H_{0, d}-H_{0, b},
$$

applying part (c) to the dual cocycle $\widetilde{V}$. Also $C E_{d-b} \supset T_{d}-T_{b}$, thus $\mathfrak{D}^{\{b, d\}}$ is a subspace of both Dom $M E_{d-b}$ and Dom $C E_{d-b}$. Therefore if $e \in S-S$ and $v \in \mathfrak{D}^{S}$ then $v e \in$ Dom $M \cap \operatorname{Dom} C$. The result follows since Dom $L=\operatorname{Dom} Z=\mathfrak{D}^{0}$.

For a cocycle $V$ and subspace $\mathrm{D}$ of $\mathrm{k}$, Corollary 1.4 permits the following definition:

$$
F^{V, \mathrm{D}}:=F^{V} \mid \mathfrak{D}_{0} \oplus(\mathfrak{D} \underline{\otimes} \mathrm{D})
$$

where $\mathfrak{D}_{0}=\mathfrak{D}^{V, 0}$ and $\mathfrak{D}=\mathfrak{D}^{V, \mathrm{D}}$. Note that for any subset $\mathrm{T}$ of $\mathrm{k}$ containing 0

$$
\mathfrak{D}^{V, T}=\mathfrak{D}^{V, \mathrm{D}} \text {, where } \mathrm{D}=\operatorname{Lin} \mathrm{T} \text {. }
$$

From the corollary we see that $\mathfrak{D}^{V,\{0, d\}} \subset$ Dom $E^{\widehat{c}} F^{V} E_{\widehat{d}}$ for all $c, d \in \mathrm{k}$, and by parts (b)(e) of the proposition,

$$
E^{\widehat{c}} F^{V} E_{\widehat{d}}=H_{c, d}^{V}-\langle c, d\rangle=G_{c, d}^{V}+\chi(c, d) \text { on } \mathfrak{D}^{V,\{d, 0\}} .
$$

For Markov-regular cocycles, that is cocycles whose Markov semigroup $Q^{0,0}$ is normcontinuous, the situation is much simpler. (See below for the definition of $\widehat{k}$ )

COROLLARY 1.5. Let $V$ be a strongly continuous left contraction cocycle on $\mathfrak{h}$ with noise dimension space $\mathrm{k}$ and suppose that one of its associated semigroups $Q^{c, d}$ is norm continuous. Then all of its associated semigroups are norm continuous and $F^{V} \in B(\mathfrak{h} \otimes \widehat{\mathrm{k}})$.

Proof. That all or none of the associated semigroups are norm continuous follows since

$$
\left\|P_{t}^{a, b}-P_{t}^{c, d}\right\|=O(\sqrt{t}) \text { for } a, b, c, d \in \mathrm{k},
$$

by (1.4). So if it $i s$ the case that all the semigroups are norm continuous then $H_{c, d}^{V} \in B(\mathfrak{h})$ for all $c, d \in \mathrm{k}$. In particular $Z^{V}=H_{0,0}^{V} \in B(\mathfrak{h})$, so that $\mathfrak{D}^{V, 0}=\mathfrak{h}$, and hence $L^{V}=T_{0}^{V}$ which is bounded by part (f) of the proposition. 
Since the semigroups associated to the dual cocycle are the adjoints of those associated with the cocycle, and must also be norm continuous, $M^{V}=T_{0}^{\widetilde{V} *} \in B(\mathfrak{h} \otimes \mathrm{k} ; \mathfrak{h})$, and from (1.8) it follows that the contraction $C^{V}$ is densely defined, hence $C^{V} \in B(\mathfrak{h} \otimes \mathrm{k})$.

Cocycle characterisation through semigroups

In Proposition 1.1 we used the family of maps $\left\{{ }^{V} Q_{t}^{c, d}: c, d \in \mathrm{T}, t \geqslant 0\right\}$ defined in terms of a given process $V$ to determine whether or not it is a left cocycle. The following result turns this around.

THEOREM $1.6\left(\left[\mathbf{L W}_{3}\right]\right)$. Let $Q_{\top}=\left\{Q^{c, d}: c, d \in \mathrm{T}\right\}$ be a family of semigroups on $\mathfrak{h}$ indexed by a total subset $\mathrm{T}$ of $\mathrm{k}$ which contains 0 . Then the following are equivalent:

(i) there is a left contraction cocycle $V$ on $\mathfrak{h}$ whose associated family of semigroups includes $Q_{\mathrm{T}}$;

(ii) for all $n \in \mathbb{N}, Y \in \mathrm{M}_{n}(|\mathfrak{h}\rangle)=B\left(\mathbb{C}^{n} ; \mathfrak{h}^{n}\right)$, and positive invertible matrices $A, B \in$ $\mathrm{M}_{n}(\mathbb{C})$, if $\left\|A^{-1 / 2} Y B^{-1 / 2}\right\| \leqslant 1$ then

$$
\left\|\left(A \bullet \varpi_{t}^{\mathbf{c}}\right)^{-1 / 2}\left(Q_{t}^{\mathbf{c}} \bullet Y\right)\left(B \bullet \varpi_{t}^{\mathbf{c}}\right)^{-1 / 2}\right\| \leqslant 1,
$$

for all $\mathbf{c} \in \mathrm{T}^{n}, t \geqslant 0$.

This requires some explanation of terms: $|\mathfrak{h}\rangle:=B(\mathbb{C} ; \mathfrak{h})$, the column operator space determined by $\mathfrak{h}([\mathbf{E f R}, \mathbf{P i s}])$; given $c, d \in \mathrm{k}, \varpi_{t}^{c, d}=\left\langle\varpi\left(c_{[0, t]}\right), \varpi\left(d_{[0, t]}\right)\right\rangle=\exp -t \chi(c, d)$, and given $\mathbf{c} \in \mathbf{k}^{n}, \varpi_{t}^{\mathbf{c}}:=\left[\varpi_{t}^{c_{i}, c_{j}}\right] \in \mathrm{M}_{n}(\mathbb{C})=B\left(\mathbb{C}^{n}\right), Q_{t}^{\mathbf{c}}:=\left[Q_{t}^{c_{i}, c_{j}}\right] \in \mathrm{M}_{n}(B(\mathfrak{h}))=B\left(\mathfrak{h}^{n}\right)$, the symbol $\bullet$ denotes the Schur product of matrices, so in particular if $T=\left[\left|u_{j}^{i}\right\rangle\right]$ then $Q_{t}^{\mathrm{c}} \bullet T=\left[\left|Q_{t}^{c_{i}, c_{j}} u_{j}^{i}\right\rangle\right] \in B\left(\mathbb{C}^{n} ; \mathfrak{h}^{n}\right)$; finally, the first matrix within each of the norms is thought of as having entries of the form $v I_{\mathfrak{h}}$ for $v \in \mathbb{C}$, thus both norms are those of $B\left(\mathbb{C}^{n} ; \mathfrak{h}^{n}\right)$.

What this result tells us is that if we can find a family of semigroups $Q_{\top}$ on $\mathfrak{h}$, indexed by a total subset $\mathrm{T}$ of $\mathrm{k}$ containing 0 , which satisfies (1.21) then there is an associated cocycle $V$ on $\mathfrak{h}$. This condition, on a putative family of semigroups $Q_{T}$, looks hard to verify. However the strength of the result lies in the fact that it is manifestly stable under pointwise limits.

THEOREM 1.7. Let $Q_{\mathrm{T}}=\left\{Q^{c, d}: c, d \in \mathrm{T}\right\}$ be a family of semigroups on $\mathfrak{h}$, indexed by a total subset $\mathrm{T}$ of $\mathrm{k}$ which includes 0 . Suppose that there is a sequence $\left(V^{(n)}\right)_{n \geqslant 1}$ of left contraction cocycles on $\mathfrak{h}$ whose associated semigroups satisfy

$$
{ }^{(n)} Q_{t}^{c, d} \rightarrow Q_{t}^{c, d} \quad \text { pointwise on } \mathfrak{h},
$$

for all $c, d \in \mathrm{T}$ and $t>0$. Then there is a unique left contraction cocycle $V$ on $\mathfrak{h}$ whose associated semigroups include the family $\mathrm{Q}_{\mathrm{T}}$. Moreover $V_{t}^{(n)} \rightarrow V_{t}$ in the weak operator topology for each $t$.

Proof. The existence of $V$ is immediate from Theorem 1.6, uniqueness follows from the totality of $\mathrm{T}$, and the convergence $V^{(n)} \rightarrow V$ is a consequence of (1.1) and contractivity.

\section{Quantum stochastic differential equations}

Let $\widehat{\mathrm{k}}:=\mathbb{C} \oplus \mathrm{k}$ and, for any subspace $\mathrm{D}$ of $\mathrm{k}$, let $\widehat{\mathrm{D}}=\mathbb{C} \oplus \mathrm{D}=\operatorname{Lin}\{\widehat{d}: d \in \mathrm{D}\}$ where $\widehat{d}:=\left(\begin{array}{l}1 \\ d\end{array}\right)$. Also let $e_{0}=\left(\begin{array}{l}1 \\ 0\end{array}\right) \in \widehat{\mathrm{k}}$ and define

$$
\Delta=I_{\mathfrak{h}} \otimes P_{\mathrm{k}}
$$

where $P_{\mathrm{k}} \in B \widehat{(\mathrm{k})}$ is the orthogonal projection with range $\left\{e_{0}\right\}^{\perp}=0 \oplus \mathrm{k}$. 
Consider now the form QSDE

$$
d V_{t}=V_{t} \mathbb{F} d \Lambda_{t}, \quad V_{0}=I,
$$

and the operator $Q S D E$

$$
d V_{t}=\widehat{V}_{t}\left(F \underline{\otimes} I_{\mathcal{F}}\right) d \Lambda_{t}, \quad V_{0}=I,
$$

for (bounded operator-valued) processes $V$ on $\mathfrak{h}$, for which we need dense subspaces $\mathfrak{D}_{0} \supset$ $\mathfrak{D}$ of $\mathfrak{h}$, and total subsets $T^{\dagger}$ and $T$ of $k$ containing 0 . Set $D=\operatorname{Lin} T$ and $D^{\dagger}=\operatorname{Lin} T^{\dagger}$.

In the first case, $\mathbb{F}$ is an operator-valued map defined on $\widehat{D^{\dagger}} \times \widehat{D}$ of the form

$$
\left(\left(\begin{array}{l}
z \\
c
\end{array}\right),\left(\begin{array}{l}
w \\
d
\end{array}\right)\right) \mapsto\left[\begin{array}{ll}
\bar{z} & 1
\end{array}\right]\left[\begin{array}{cc}
K & M_{d} \\
L^{c} & N_{d}^{c}
\end{array}\right]\left[\begin{array}{c}
w \\
1
\end{array}\right]=\bar{z} w K+\bar{z} M_{d}+w L^{c}+N_{d}^{c}
$$

where $K \in \mathcal{O}\left(\mathfrak{D}_{0}\right), c \mapsto L^{c}$ is conjugate linear $\mathrm{D}^{\dagger} \rightarrow \mathcal{O}\left(\mathfrak{D}_{0}\right), d \mapsto M_{d}$ is linear $\mathrm{D} \rightarrow \mathcal{O}(\mathfrak{D})$ and $(c, d) \mapsto N_{d}^{c}$ is sesquilinear $\mathrm{D}^{\dagger} \times \mathrm{D} \rightarrow \mathcal{O}(\mathfrak{D})$ (thus $\mathbb{F}\left(\left(\begin{array}{l}z \\ c\end{array}\right),\left(\begin{array}{l}w \\ d\end{array}\right)\right) \in \mathcal{O}(\mathfrak{D})$ in general, and is $\mathcal{O}\left(\mathfrak{D}_{0}\right)$-valued if $\left.d=0\right)$, and $V$ is a T'-T-solution of (2.1) on $\mathfrak{D}_{0} \otimes \varepsilon(0)+\mathfrak{D} \otimes \mathcal{E}_{\mathrm{T}}$ if, in the notation $\widehat{g}(s):=\widehat{g(s)}$,

$$
\left\langle u \varepsilon(f),\left(V_{t}-I\right) v \varepsilon(g)\right\rangle=\int_{0}^{t}\left\langle u \varepsilon(f), V_{s} \mathbb{F}(\widehat{f}(s), \widehat{g}(s)) v \varepsilon(g)\right\rangle d s
$$

for all $u \in \mathfrak{h}, f \in \mathbb{S}_{T^{+}},(v, g) \in\left(\mathfrak{D}_{0} \times\{0\}\right) \cup\left(\mathfrak{D} \times \mathbb{S}_{\mathrm{T}}\right)$ and $t \geqslant 0$. In particular $V$ is weakly continuous in an obvious sense.

In the second case $F \in \mathcal{O}\left(\mathfrak{D}_{0} \oplus(\mathfrak{D} \underline{\otimes D})\right), \widehat{V}_{t}$ stands for the operator on $\mathfrak{h} \otimes \widehat{\mathfrak{k}} \otimes \mathcal{F}$ obtained from $V_{t} \otimes I_{\widehat{\mathrm{k}}}$ by tensor flipping, and there are two basic kinds of solution: $V$ is a $\mathrm{T}^{\dagger}$-weak solution of $(2 \cdot 2)$ on $\mathfrak{D}_{0} \otimes \varepsilon(0)+\mathfrak{D} \otimes \mathcal{E}_{\mathrm{T}}$ if (2.3) holds for the component map of $F$, defined by

$$
\mathbb{F}(\xi, \eta)=E^{\xi} F E_{\eta}, \quad \xi \in \widehat{\mathrm{D}^{\dagger}}, \eta \in \widehat{\mathrm{D}} .
$$

In other words, setting $\zeta(s)=v e_{0} \varepsilon(0)+w \widehat{g}(t) \varepsilon(g)$,

$$
\begin{aligned}
\langle u \varepsilon(f) & \left.,\left(V_{t}-I\right)\{v \varepsilon(0)+w \varepsilon(g)\}\right\rangle \\
= & \int_{0}^{t}\left\langle u \varepsilon(f), V_{s} E^{\widehat{f}(s)} F \underline{\otimes} I_{\mathcal{F}} \zeta(s)\right\rangle d s \\
= & \int_{0}^{t}\left\langle u \varepsilon(f), V_{s}\left\{\left(K+L^{f(s)}\right) v \varepsilon(0)+\left(K+L^{f(s)}+M_{g(s)}+N_{g(s)}^{f(s)}\right) w \varepsilon(g)\right\}\right\rangle d s,
\end{aligned}
$$

where $F=\left[\begin{array}{cc}K & M \\ L & N\end{array}\right]$ in block matrix form, and $L^{c}=E^{c} L$ etc.

$V$ is a strong solution of $(2 \cdot 2)$ on the same domain if the map

$$
t \longmapsto\left\|\widehat{V}_{t} \Delta F \underline{\otimes} I_{\mathcal{F}} \zeta(t)\right\|^{2}+\left\|\widehat{V}_{t} \Delta^{\perp} F \underline{\otimes} I_{\mathcal{F}} \zeta(t)\right\|
$$

is locally integrable for each $v \in \mathfrak{D}_{0}, w \in \mathfrak{D}$, and $g \in \mathbb{S}_{\mathrm{T}}, V$ is strongly measurable, (and so $\widehat{V} F \otimes I_{\mathcal{F}}$ is stochastically integrable), and if it satisfies the quantum stochastic integral equation

$$
V_{t}=I+\int_{0}^{t} \widehat{V}_{s}\left(F \underline{\otimes} I_{\mathcal{F}}\right) d \Lambda_{s} .
$$

In particular $V$ is then strongly continuous on its domain ([L]), hence on all of $\mathfrak{h} \otimes \mathcal{F}$ by contractivity; the First Fundamental Formula of quantum stochastic calculus implies that $V$ is necessarily a k-weak solution on $\mathfrak{D}_{0} \otimes \varepsilon(0)+\mathfrak{D} \underline{\otimes} \mathcal{E}_{\mathrm{T}}$. 
We recall the basic implication for $F$ of contractivity of a strong solution of (2.2), and include its short proof for the convenience of the reader (cf. [FaW]).

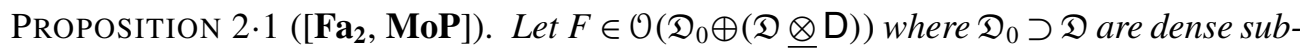
spaces of $\mathfrak{h}$ and $\mathrm{D}=\mathrm{Lin} \mathrm{T}$ for a total subset $\mathrm{T}$ of $\mathrm{k}$ containing 0 , and suppose that (2.2) has a strong contractive solution on $\mathfrak{D}_{0} \otimes \varepsilon(0)+\mathfrak{D} \otimes \mathcal{E}_{\mathrm{T}}$. Then $F$ satisfies the form inequality

$$
2 \operatorname{Re}\langle\xi, F \xi\rangle+\|\Delta F \xi\|^{2} \leqslant 0
$$

with equality if the solution is isometric.

Proof. Let $\xi \in \mathfrak{D}_{0} \oplus(\mathfrak{D} \underline{\otimes D})$. Then $\xi$ is expressible in the form $u_{0} e_{0}+\sum_{i=1}^{n} u_{i} \widehat{c_{i}}$ for some $u_{0} \in \mathfrak{D}_{0}, n \in \mathbb{N}, u_{1}, \ldots, u_{n} \in \mathfrak{D}$ and $c_{1}, \ldots, c_{n} \in \mathrm{T}$. Let $\zeta=u_{0} \varepsilon(0)+\sum_{i=1}^{n} u_{i} \varepsilon\left(f_{i}\right)$ and $\zeta(s)=u_{0} e_{0} \varepsilon(0)+\sum_{i=1}^{n} u_{i} \widehat{f_{i}}(s) \varepsilon\left(f_{i}\right)$ where $f_{i}=c_{i[0, T[}$ for $i=1, \ldots, n$ and some $T>0$. Then, by the Second Fundamental Formula of quantum stochastic calculus,

$$
\begin{aligned}
0 & \geqslant t^{-1}\left(\left\|V_{t} \zeta\right\|^{2}-\|\zeta\|^{2}\right) \\
& =t^{-1} \int_{0}^{t}\left\{2 \operatorname{Re}\left\langle\widehat{V}_{s} \zeta(s), \widehat{V}_{s}\left(F \underline{\otimes} I_{\mathcal{F}}\right) \zeta(s)\right\rangle+\left\|\widehat{V}_{s}\left(\Delta F \underline{\otimes} I_{\mathcal{F}}\right) \zeta(s)\right\|^{2}\right\} d s
\end{aligned}
$$

for $t \in] 0, T]$, with equality if $V$ is isometric. Using the continuity of the integrand at the origin, letting $t \rightarrow 0$ and then letting $T \rightarrow 0$ now gives the result.

Remarks. (i) If it is assumed further that all the T-components $\left(E^{\widehat{c}} F E_{\widehat{d}}: c, d \in \mathrm{T}\right)$ are bounded, then (2.2) may be solved by Picard iteration and Mohari and Fagnola showed that (2.4) is also sufficient for contractivity of the solution. In fact boundedness of Tcomponents and contractivity of the solution implies that $F$ itself is bounded ([LW $\mathbf{L}$, theorem 7.5]) so that (2.4) simplifies to the operator inequality

$$
F+F^{*}+F^{*} \Delta F \leqslant 0 .
$$

The solution is also unique amongst $\mathrm{T}^{\dagger}$-weak solutions (cf. Theorem $2 \cdot 3$ below).

(ii) Since the integrability condition for being a strong solution is automatically satisfied by strongly measurable contraction processes, any strongly measurable weak solution of $(2 \cdot 2)$ is necessarily a strong solution on the same domain.

We next show how the assumption of strong measurability also renders form solutions into strong operator solutions.

THEOREM 2.2. Let $V$ be a strongly measurable contraction process on $\mathfrak{h}$ with noise dimension space $\mathrm{k}$, let $\mathrm{T}$ be a total subset of $\mathrm{k}$ containing 0 and let $\mathfrak{D}_{0} \supset \mathfrak{D}$ be dense subspaces of $\mathfrak{h}$. Set $\mathrm{D}=\mathrm{Lin} \mathrm{T}$, and assume that $\mathrm{D}$ has an orthonormal basis. If $V$ is a T-T-solution of the form $\mathrm{QSDE}(2 \cdot 1)$ on $\mathfrak{D}_{0} \otimes \varepsilon(0)+\mathfrak{D} \otimes \mathcal{E}_{\mathrm{T}}$, and each map $\xi \mapsto \mathbb{F}(\xi, \eta) v$ is continuous then $\mathbb{F}$ is the component map of an operator $F \in \mathcal{O}\left(\mathfrak{D}_{0} \oplus(\mathfrak{D} \otimes \mathbb{D})\right)$ and $V$ satisfies the corresponding operator QSDE (2.2) strongly on the same domain.

Proof. By the second remark above it suffices to show that $\mathbb{F}$ is necessarily the component map of an operator $F \in \mathcal{O}\left(\mathfrak{D}_{0} \oplus(\mathfrak{D} \underline{\otimes} \mathrm{D})\right)$. For any subspace $\mathrm{h}$ of $\mathrm{k}$ of the form Lin $\mathrm{T}_{0}$ where $\mathrm{T}_{0}$ is a finite subset of $\mathrm{T}$, define an operator $F^{\mathrm{h}} \in \mathcal{O}\left(\mathfrak{D}_{0} \oplus(\mathfrak{D} \underline{\otimes} \mathrm{h})\right)$ by

$$
F^{\mathrm{h}}=\sum_{\alpha, \beta} E_{e_{\alpha}} \mathbb{F}\left(e_{\alpha}, e_{\beta}\right) E^{e_{\beta}}
$$


where $\left(e_{\alpha}\right)$ is an orthonormal basis for $\widehat{h}$ which includes the vector $e_{0}=\left(\begin{array}{l}1 \\ 0\end{array}\right)$. By sesquilinearity, $F^{\mathrm{h}}$ does not depend on the choice of basis and, for $\xi \in \widehat{\mathrm{h}}$ and $(v, \eta) \in\left(\mathfrak{D}_{0} \times\right.$ $\left.\mathbb{C} e_{0}\right) \cup(\mathfrak{D} \times \widehat{\mathrm{h}})$,

$$
E^{\xi} F^{\mathrm{h}} v \eta=\mathbb{F}(\xi, \eta) v
$$

Also let $J^{\text {h }}$ be the natural isometric embedding $\mathfrak{h} \otimes \Gamma\left(L^{2}\left(\mathbb{R}_{+} ; \mathfrak{h}\right)\right) \rightarrow \mathfrak{h} \otimes \mathcal{F}$, obtained by second quantisation of the inclusion map $\mathrm{h} \rightarrow \mathrm{k}$. Then it is easily verified that the process $V^{\mathrm{h}}:=\left(J^{\mathrm{h} *} V_{t} J^{\mathrm{h}}\right)_{t \geqslant 0}$ satisfies the QSDE $d X_{t}=\widehat{X}_{t}\left(F^{\mathrm{h}} \otimes I\right) d \Lambda_{t}, X_{0}=I, \mathrm{~T}_{0}$-weakly on $\mathfrak{D}_{0} \otimes$ $\varepsilon(0)+\mathfrak{D} \otimes \mathcal{E}_{\mathrm{T}_{0}}$. Since $V^{\mathrm{h}}$ is contractive and strongly measurable it satisfies the equation strongly. Therefore, by Proposition $2 \cdot 1, F^{\mathrm{h}}$ satisfies

$$
\left\|\Delta F^{\mathrm{h}} v \eta\right\|^{2} \leqslant-2 \operatorname{Re}\left\langle v \eta, F^{\mathrm{h}} v \eta\right\rangle=-2 \operatorname{Re}\langle v, \mathbb{F}(\eta, \eta) v\rangle
$$

for $(v, \eta) \in\left(\mathfrak{D}_{0} \times \mathbb{C} e_{0}\right) \cup(\mathfrak{D} \times \widehat{\mathrm{h}})$.

Now let $\left(d_{i}\right)_{i \in I}$ be an orthonormal basis for $\mathrm{k}$ taken from $\mathrm{D}$ (with $0 \notin I$ ), and set $\widehat{I}=$ $\{0\} \cup I, e_{0}=\left(\begin{array}{l}1 \\ 0\end{array}\right)$ and $e_{i}=\left(\begin{array}{l}0 \\ d_{i}\end{array}\right)$ so that $\left(e_{\alpha}\right)_{\alpha \in \hat{I}}$ is an orthonormal basis for $\widehat{\mathrm{k}}$. Then, for $(v, \eta) \in\left(\mathfrak{D} \times \mathbb{C}\left(\begin{array}{l}0 \\ d\end{array}\right)\right) \cup\left(\mathfrak{D}_{0} \times \mathbb{C} e_{0}\right)$ where $d \in \mathrm{T}$ and $I_{0}$ a finite subset of $I$, applying (2.6) with $\mathrm{h}=\operatorname{Lin}\left(\{d\} \bigcup\left\{d_{i}: i \in I_{0}\right\}\right)$ gives

$$
\begin{aligned}
\sum_{i \in I_{0}}\left\|\mathbb{F}\left(e_{i}, \eta\right) v\right\|^{2} & =\left\|\sum_{i \in I_{0}} E_{e_{i}} E^{e_{i}} F^{\mathrm{h}} v \eta\right\|^{2} \\
& \leqslant\left\|\Delta F^{\mathrm{h}} v \eta\right\|^{2} \leqslant-2 \operatorname{Re}\langle v, \mathbb{F}(\eta, \eta) v\rangle
\end{aligned}
$$

and so the orthogonal sum $\sum_{i \in I} E_{e_{i}} \mathbb{F}\left(e_{i}, \eta\right) v$ is convergent. Thus an operator $F \in \mathcal{O}\left(\mathfrak{D}_{0} \oplus\right.$ $(\mathfrak{D} \otimes \mathrm{D}))$ is defined by linear extension of the prescription

$$
v \eta \longmapsto \sum_{\alpha \in \widehat{I}} E_{e_{\alpha}} \mathbb{F}\left(e_{\alpha}, \eta\right) v .
$$

By the continuity assumption on $\mathbb{F}, E^{\xi} F v \eta=\mathbb{F}(\xi, \eta) v$ for $\xi \in \widehat{\mathrm{D}}$ and $v \eta$ as above, and it follows that $F$ is independent of the choice of basis $\left(d_{i}\right)_{i \in I}$, hence is the component map of $\mathbb{F}: E^{\xi} F E_{\eta}=\mathbb{F}(\xi, \eta)$ for $\xi, \eta \in \widehat{\mathrm{D}}$. This completes the proof.

Remarks. Dixmier showed that a pre-Hilbert space need not have an orthonormal basis ([Bou, V.70]); however the assumption on D is automatically satisfied if either the Hilbert space $\mathrm{k}$ is separable or the set $\mathrm{T}$ contains a subset which is orthogonal and total.

Since contraction processes satisfying the form QSDE are weakly continuous, the strong measurability assumption is redundant when $\mathfrak{h}$ and $\mathrm{k}$ are both separable or, in view of Lemma 1.2, when the solution is a left cocycle.

This connects with issues of uniqueness.

THEOREM 2.3. Let $\mathbb{F}$ be a sesquilinear map $\widehat{\mathrm{D}^{\dagger}} \times \widehat{\mathrm{D}} \rightarrow \mathcal{O}(\mathfrak{D})$, where $\mathfrak{D}$ is a dense subspace of $\mathfrak{h}, \mathrm{D}^{\dagger}=$ Lin $\mathrm{T}^{\dagger}$ and $\mathrm{D}=\mathrm{Lin} \mathrm{T}$ for total subsets $\mathrm{T}^{\dagger}$ and $\mathrm{T}$ of $\mathrm{k}$ that contain 0.

(a) Suppose that $\mathrm{T}^{\dagger}=\mathbb{R} \mathrm{T}^{\dagger}$ and $\mathrm{T}=\mathbb{R T}$. If $K:=\mathbb{F}\left(e_{0}, e_{0}\right)$ is a pregenerator of a $C_{0^{-}}$ contraction semigroup on $\mathfrak{h}$ then the form QSDE (2.1) has at most one contractive $\mathrm{T}^{\dagger}$-T-solution on $\mathfrak{D} \otimes \mathcal{E}_{\mathrm{T}}$.

(b) If the form QSDE $(2 \cdot 1)$ has a unique contractive $\mathrm{T}^{\dagger}$-T-solution $V$ on $\mathfrak{D} \otimes \mathcal{E}_{\mathrm{T}}$ then $V$ is a left contraction cocycle.

(c) If the form QSDE $(2 \cdot 1)$ has a $\mathrm{T}^{\dagger}$-T-solution $V$ on $\mathfrak{D} \otimes \mathcal{E}_{\mathrm{T}}$ which is a left contraction cocycle then $\mathfrak{D} \subset \mathfrak{D}^{V, \mathrm{D}}$ and $\left.E^{\widehat{c}} F^{V} E_{\widehat{d}}\right|_{\mathfrak{D}}=\mathbb{F}(\widehat{c}, \widehat{d})$ for all $c \in \mathrm{D}^{\dagger}, d \in \mathrm{D}$. 
Part (a) is Mohari's Uniqueness Theorem ([Moh]). The invariance of $\mathrm{T}$ (and $\mathrm{T}^{\dagger}$ ) under scalar multiplication can be weakened to the following:

$$
\text { for each } d \in \mathrm{T} \text { there exists } \epsilon>0 \text { such that }[0, \epsilon] d \subset \mathrm{T} \text {, }
$$

which is sufficiently strong to allow differentiation at a crucial stage in his argument. It is clear from Meyer's treatment ([Mey, page 191]) that the result remains valid in this generality. Part (b) is proved by verifying that, for each $t>0$,

$$
V_{s}^{t}= \begin{cases}V_{s} & \text { if } s \leqslant t \\ V_{t} \sigma_{t}\left(V_{s-t}\right) & \text { if } s>t\end{cases}
$$

defines a contraction process $V^{t}$ which also satisfies $(2 \cdot 1)$. This is easily checked by treating $\mathfrak{h} \otimes \mathcal{F}_{[0, t[}$ as an initial space and using the explicit action of shifts on exponential vectors.

Proof of part (c). Pick $u \in \mathfrak{h}, v \in \mathfrak{D}, c \in \mathrm{T}^{\dagger}$ and $d \in \mathrm{T}$, and set $f=c_{[0,1[}$ and $g=d_{[0,1[}$. Then for all $0<t<1$

$$
\left\langle u \varepsilon(f),\left(V_{t}-I\right) v \varepsilon(g)\right\rangle=\int_{0}^{t}\left\langle u \varepsilon(f), V_{s} \mathbb{F}(\widehat{c}, \widehat{d}) v \varepsilon(g)\right\rangle d s .
$$

But, for the same $t$,

$$
\left\langle u,\left(P_{t}^{c, d}-I\right) v\right\rangle=\left\langle u \varepsilon(f),\left(V_{t}-I\right) v \varepsilon(g)\right\rangle e^{(t-1)\langle c, d\rangle}+\left(e^{t\langle c, d\rangle}-1\right)\langle c, d\rangle\langle u, v\rangle
$$

and consequently

$$
\lim _{t \rightarrow 0} t^{-1}\left\langle u,\left(P_{t}^{c, d}-I\right) v\right\rangle=\langle u,(\mathbb{F}(\widehat{c}, \widehat{d})-\langle c, d\rangle) v\rangle .
$$

Since this holds for all $u \in \mathfrak{h}$ it follows that $v \in \operatorname{Dom} H_{c, d}^{V}$ and that $\mathbb{F}(\widehat{c}, \widehat{d})-\langle c, d\rangle \subset H_{c, d}^{V}$ for all $c \in \mathrm{D}^{\dagger}$ and $d \in \mathrm{D}$ ([Dav, theorem 1.24]). Hence $\mathfrak{D} \subset \mathfrak{D}^{V, \mathrm{D}}$ and so $E^{\widehat{c}} F^{V} E_{\widehat{d}} \mid \mathfrak{D}=$ $\mathbb{F}(\widehat{c}, \widehat{d})$ by $(1 \cdot 20)$.

\section{Necessary conditions for contractive solution}

In this section we explore necessary conditions on $F$ for the existence of contractive solutions of (2.2). Recall that densely defined dissipative operators are closable with dissipative closures.

Proposition 3·1. Let $F \in \mathcal{O}\left(\mathfrak{D}_{0} \oplus(\mathfrak{D} \otimes \mathrm{D})\right)$, for dense subspaces $\mathfrak{D}_{0} \supset \mathfrak{D}$ of $\mathfrak{h}$ and $\mathrm{D}$ of $\mathrm{k}$, have block matrix form $\left[\begin{array}{cc}K & M \\ L & C-I\end{array}\right]$ and satisfy the form inequality (2.4). For each $c \in \mathrm{k}$ and $d \in \mathrm{D}$ define $G_{c, d}^{0}:=E^{\widehat{c}} F E_{\widehat{d}}-\chi(c, d)$. Then:

(a) $C$ is a contraction and, for all $u \in \mathfrak{D}_{0}$,

$$
\|L u\|^{2}+2 \operatorname{Re}\langle u, K u\rangle \leqslant 0
$$

(b) $F$ and $G_{c, d}^{0}$ are dissipative; let $G_{c, d}=\overline{G_{c, d}^{0}}$ and $Z=G_{0,0}=\bar{K}$;

(c) $L$ is $G_{c, d}^{0}$-bounded, with relative bound 0 , on Dom $G_{c, d}^{0}$;

(d) for each $a \in \mathrm{k}, G_{a, d}^{0}$ is a relatively bounded perturbation of $G_{c, d}^{0}$ with relative bound 0 , Dom $G_{a, d}=\operatorname{Dom} G_{c, d}$, and $G_{a, d}$ is a $C_{0}$-semigroup generator if and only if $G_{c, d}$ is;

(e) Dom $M^{*} \supset$ Dom $K$ and, for all $u \in \mathfrak{D}_{0}$,

$$
\left\|\left(L+C M^{*}\right) u\right\|^{2}+\left\|M^{*} u\right\|^{2}+2 \operatorname{Re}\langle u, K u\rangle \leqslant 0,
$$

in particular, $M^{*}$ is $K$-bounded with relative bound 0 ; 
(f) $\bar{F}$ also satisfies (2.4), moreover $\bar{F} \supset F^{\prime}$ where $F^{\prime}=\left[\begin{array}{cc}Z & \bar{M} \\ L^{\prime} & \bar{C}-I\end{array}\right], L^{\prime}$ being the continuous extension (in the graph norm of $Z$ ) of $L$ to Dom $Z$.

Proof. For $\xi=\left(\begin{array}{l}u \\ \eta\end{array}\right) \in \mathfrak{D}_{0} \oplus(\mathfrak{D} \underline{\otimes} \mathrm{D}),(2 \cdot 4)$ is equivalent to

$$
\|L u+C \eta\|^{2} \leqslant-2 \operatorname{Re}\langle u, K u+M \eta\rangle+\|\eta\|^{2},
$$

and setting $u=0$, respectively $\eta=0$, shows that (a) holds. Now abbreviate $E^{c} L, M E_{d}$ and $E^{c} C E_{d}$ to $L^{c}, M_{d}$ and $C_{d}^{c}$ respectively, and denote Dom $G_{c, d}^{0}$ by $\mathfrak{D}_{d}^{0}$, thus

$$
G_{c, d}^{0}=K+L^{c}+M_{d}+C_{d}^{c}-\frac{1}{2}\|c\|^{2}-\frac{1}{2}\|d\|^{2},
$$

where $\mathfrak{D}_{d}^{0}=\mathfrak{D}_{0}$ if $d=0$ and equals $\mathfrak{D}$ otherwise.

If $\eta=u d$ where $d \in \mathrm{D}$ and $u \in \mathfrak{D}_{d}^{0}$ then (3.3) reads

$$
\left\|\left(L+C_{d}\right) u\right\|^{2} \leqslant-2 \operatorname{Re}\left\langle u,\left(K+M_{d}-\frac{1}{2}\|d\|^{2}\right) u\right\rangle,
$$

so, for $c \in \mathrm{k}$ and $d \in \mathrm{D}$,

$$
\begin{aligned}
-2 \operatorname{Re}\left\langle u, G_{c, d}^{0} u\right\rangle & =-2 \operatorname{Re}\left\langle u,\left\{\left(K+M_{d}-\frac{1}{2}\|d\|^{2}\right)+\left(L^{c}+C_{d}^{c}-\frac{1}{2}\|c\|^{2}\right)\right\} u\right\rangle \\
& \geqslant\left\|\left(L+C_{d}\right) u\right\|^{2}-2 \operatorname{Re}\left\langle u c,\left(L+C_{d}\right) u\right\rangle+\|u c\|^{2} \\
& =\left\|\left(L+C_{d}-E_{c}\right) u\right\|^{2}
\end{aligned}
$$

for all $u \in \mathfrak{D}_{d}^{0}$. Thus $G_{c, d}^{0}$ is dissipative, moreover

$$
\left\|\left(L+C_{d}-E_{c}\right) u\right\| \leqslant \sqrt{-2 \operatorname{Re}\left\langle\lambda^{-1} u, \lambda G_{c, d}^{0} u\right\rangle} \leqslant \lambda\left\|G_{c, d}^{0} u\right\|+\lambda^{-1}\|u\|
$$

for all $\lambda>0$ and so, since $\left(C_{d}-E_{c}\right)$ is bounded, $L$ is $G_{c, d}^{0}$-bounded with relative bound 0 . Since $F$ is clearly dissipative, we have established (b) and (c). Since $G_{a, d}^{0}-G_{c, d}^{0}=$ $E^{a-c} L+C_{d}^{a-c}+\frac{1}{2}\left(\|c\|^{2}-\|a\|^{2}\right)$ it also follows that $G_{a, d}^{0}$ is a relatively bounded perturbation of $G_{c, d}^{0}$ with relative bound 0 , and so (d) follows from Gustafson's Theorem.

Now let $u \in \mathfrak{D}_{0}$. Then, from (3.3), $2|\langle u, M \eta\rangle| \leqslant\|\eta\|^{2}-2 \operatorname{Re}\langle u, K u\rangle$ for each $\eta \in$ $\mathfrak{D} \otimes D$. This implies that $u \in$ Dom $M^{*}$. Thus Dom $M^{*} \supset \mathfrak{D}_{0}$ and (3.3) reads $\|L u+C \eta\|^{2}+$ $2 \overline{\operatorname{Re}}\left\langle M^{*} u, \eta\right\rangle+2 \operatorname{Re}\langle u, K u\rangle \leqslant\|\eta\|^{2}$, now valid for $u \in \mathfrak{D}_{0}$ and $\eta \in \mathfrak{h} \otimes \mathrm{k}$. Putting $\eta=M^{*} u$ gives (3.2), in particular

$$
\left\|M^{*} u\right\|^{2} \leqslant 2|\langle u, K u\rangle| \leqslant\left(\lambda\|K u\|+\lambda^{-1}\|u\|\right)^{2}
$$

for $\lambda>0$, showing that (e) holds. Since $F$ is densely defined and dissipative it is closable and it is easily verified that its closure contains $F^{\prime}$, and that it inherits the property (2.4) from $F$. Thus (f) holds too and the proof is complete.

Remarks. (i) The form inequality (2.4) is therefore equivalent to (3.1) together with contractivity of $C$, the inclusion Dom $M^{*} \supset \mathfrak{D}_{0}$ and the following inequality holding for $u \in \mathfrak{D}_{0}$ and $\eta \in \mathfrak{h} \otimes \mathbf{k}:$

$$
\left|\left\langle\left(M^{*}+C^{*} L\right) u, \eta\right\rangle\right|^{2} \leqslant\left(2 \operatorname{Re}\langle u,(-K) u\rangle-\|L u\|^{2}\right)\left(\|\eta\|^{2}-\|C \eta\|^{2}\right) .
$$

If equality holds in (2.4) then $C$ is isometric and $\|L u\|^{2}+2 \operatorname{Re}\langle u, K u\rangle=0$ for all $u \in \mathfrak{D}_{0}$, in turn, if either of these conditions hold then $M^{*} \supset-C^{*} L$.

(ii) In view of (f), the proposition still holds if $K, L$ and $C$ are replaced by $Z, L^{\prime}$ and $\bar{C}$ respectively, and $M$ is replaced by the restriction of $\bar{M}$ to any dense subspace of its domain of the form $\mathfrak{D}_{1}^{\prime} \underline{\otimes} \mathrm{D}^{\prime}$. 
Proposition 3.2. Let $F$ and $F^{\prime}$ be as in Proposition 3.1. Suppose that $Z$ is a generator of a $C_{0}$-semigroup and let $F^{(n)}:=I^{(n) *} \bar{F} I^{(n)}$, where $I^{(n)}=\operatorname{diag}\left[J^{(n)}, I_{\mathfrak{h} \otimes \mathfrak{k}}\right] \in B(\mathfrak{h} \otimes \widehat{\mathrm{k}})$, $J^{(n)}$ being the contraction $\left(I-n^{-1} Z\right)^{-1}$. Then $F^{(n)}$ is bounded and its closure satisfies the operator inequality $(2 \cdot 5)$, and $F^{(n)} \rightarrow F$ pointwise on $\mathfrak{D}_{0} \oplus(\mathfrak{D} \underline{\otimes D})$.

Proof. Note first that $I^{(n)}$ leaves Dom $F^{\prime}$ invariant, and that $\Delta I^{(n)}=\Delta$. Thus for $\xi \in$ Dom $F$, putting $\xi_{n}=I^{(n)} \xi$,

$$
2 \operatorname{Re}\left\langle\xi, F^{(n)} \xi\right\rangle+\left\|\Delta F^{(n)} \xi\right\|^{2}=2 \operatorname{Re}\left\langle\xi_{n}, \bar{F} \xi_{n}\right\rangle+\left\|\Delta \bar{F} \xi_{n}\right\|^{2} \leqslant 0,
$$

by Proposition $3 \cdot 1$, thus $F^{(n)}$ satisfies the form inequality $(2 \cdot 4)$. Now let $\left[\begin{array}{ll}K^{(n)} & M^{(n)} \\ L^{(n)} & \frac{\bar{C}}{-I}\end{array}\right]$ be the block matrix form of $F^{(n)}$. Since $K^{(n)}=J^{(n) *} Z J^{(n)} \in B(\mathfrak{h})$ it follows from Proposition 3.1 that $L^{(n)}$ and $M^{(n) *}$ are bounded, and so $F^{(n)}$ is bounded, hence extends to $\mathfrak{h} \otimes \widehat{\mathrm{k}}$. Thus $F^{(n)}$ satisfies the operator inequality $(2 \cdot 5)$. Now $\left(J^{(n)}\right)$ and $\left(J^{(n) *}\right)$ are sequences of contractions which converge strongly to $I$ and, for $v \in \operatorname{Dom} Z, J^{(n)} v \rightarrow v$ in the graph norm of $Z$. Thus $K^{(n)} \rightarrow Z$ on $\mathfrak{D}_{0}, L^{(n)}=L^{\prime} J^{(n)} \rightarrow L$ on $\mathfrak{D}_{0}$ (since $L^{\prime}$ is $Z$-bounded) and $M^{(n)}=$ $J^{(n) *} \bar{M} \rightarrow M$ on $\mathfrak{D} \underline{\otimes}$. In other words $F^{(n)} \rightarrow F$ pointwise on $\mathfrak{D}_{0} \oplus(\mathfrak{D} \underline{\otimes} \mathrm{D})$.

\section{Stochastic Hille-Yosida}

In this section we obtain the stochastic generator of a strongly continuous left contraction cocycle - when it has one; an existence theorem for the QSDE (2.2) is established; and some examples are discussed. We also briefly describe the situation when $\mathrm{k}$ is separable and has a given orthonormal basis.

\section{Stochastic generator of a cocycle}

We first show that strongly continuous left contraction cocycles satisfy a quantum stochastic differential equation under a minimal condition for the equation to make sense namely that there is an available dense domain for a coefficient operator to act on. It amounts to a weak-differentiability condition (cf. [AJL, Fa $\mathbf{A}_{2}$ ]). Recall the notation (1.18) and the identities $(1 \cdot 19)$ and $(1 \cdot 20)$.

THEOREM 4.1. Let $V$ be a strongly continuous left contraction cocycle on $\mathfrak{h}$ with noise dimension space $\mathrm{k}$, let $\mathrm{T}^{\dagger}$ and $\mathrm{T}$ be total subsets of $\mathrm{k}$ containing 0 , and let $\mathrm{D}=\mathrm{Lin} \mathrm{T}$, $\mathrm{D}^{\dagger}=\operatorname{Lin} \mathrm{T}^{\dagger}$ and $Z=H_{0,0}^{V}$. If $\mathfrak{D}^{V, \top}$ is dense in $\mathfrak{h}$ then the following hold.

(a) For $F=F^{V, T}$, the process $V$ satisfies the operator QSDE (2.2) strongly on $\mathfrak{D}^{V, 0} \otimes$ $\varepsilon(0)+\mathfrak{D}^{V, T} \otimes \mathcal{E}_{\mathrm{D}}$.

(b) If $\mathfrak{D}$ is a core for $Z$ contained in $\mathfrak{D}^{V, T}$, then $V$ is the unique contractive $\mathrm{D}^{\dagger}$-weak solution of $(2 \cdot 2)$ on $\mathfrak{D} \otimes \mathcal{E}_{\mathrm{D}}$, for $F=\left.F^{V, \mathrm{~T}}\right|_{\mathfrak{D} \otimes \widehat{\mathrm{D}}}$.

(c) If $\mathfrak{D}^{\widetilde{V}, T^{\dagger}}$ is also dense in $\mathfrak{h}$ then $\left(F^{V, \boldsymbol{T}}\right)^{*} \supset F^{\widetilde{V}}, \widetilde{T}^{\dagger}$.

Proof. (a) Since $V$ is strongly measurable and contractive it suffices to show that $V$ is a k-weak solution by the second remark after Proposition $2 \cdot 1$. But this follows from the semigroup representation as follows. Let $u \in \mathfrak{h}, f \in \mathbb{S}$ and $(v, g) \in\left(\mathfrak{D}^{V, 0} \times\{0\}\right) \cup\left(\mathfrak{D}^{V, \mathrm{D}} \times\right.$ $\left.\mathbb{S}_{\mathrm{D}}\right)$. Then by adaptedness and the semigroup representation $(1 \cdot 1)$,

$$
\left\langle u \varepsilon(f), V_{t} v \varepsilon(g)\right\rangle=\left\langle u, P_{t_{1}-t_{0}}^{f\left(t_{0}\right), g\left(t_{0}\right)} \cdots P_{t-t_{n}}^{f\left(t_{n}\right), g\left(t_{n}\right)} v\right\rangle e^{\int_{t}^{\infty}\langle f(s), g(s)\rangle d s},
$$

and since $\mathfrak{D}^{V, \mathrm{D}} \subset$ Dom $H_{c, d}^{V}$ for all $c \in \mathrm{k}$ and $d \in \mathrm{D}$ by Corollary 1.4 , the (a.e.) derivative of this with respect to $t$ is

$$
\left\langle u, P_{t_{1}-t_{0}}^{f\left(t_{0}\right), g\left(t_{0}\right)} \cdots P_{t-t_{n}}^{f\left(t_{n}\right), g\left(t_{n}\right)}\left(H_{f\left(t_{n}\right), g\left(t_{n}\right)}-\langle f(t), g(t)\rangle\right) v\right\rangle e^{\int_{t}^{\infty}\langle f(s), g(s)\rangle d s},
$$


in other words $\left\langle u \varepsilon(f), V_{t} E^{\widehat{f}(t)} F E_{\widehat{g}(t)} v \varepsilon(g)\right\rangle$ by (1.20). Thus $V$ satisfies (2.2) k-weakly on $\mathfrak{D}^{V, 0} \otimes \varepsilon(0)+\mathfrak{D}^{V, \mathrm{D}} \otimes \mathcal{E}_{\mathrm{D}}$.

(b) This follows from Theorem $2 \cdot 3$.

(c) This follows from $(1 \cdot 16):\left(F^{V, T}\right)^{*} \supset\left(F^{V}\right)^{*} \supset F^{\widetilde{V}} \supset F^{\widetilde{V}, \mathbf{T}^{\dagger}}$.

Remark. By Corollary 1.5 if $V$ is Markov-regular then $F^{V} \in B(\mathfrak{h} \otimes \widehat{\mathrm{k}})$, so $\mathfrak{D}^{V, \mathrm{k}}=\mathfrak{h}$ and hence $V$ satisfies the operator QSDE on $\mathfrak{h} \otimes \mathcal{E}_{\mathrm{k}}$ for this bounded operator - this is theorem $6 \cdot 7$ of $\left[\mathbf{L W}_{2}\right]$.

The theorem also extends the main result of [AJL] to infinite dimensional noise. Note that an application of the Banach-Steinhaus Theorem is needed there in order to show that the form QSDE coefficient is actually the component map of an operator. In infinite dimensions the same argument again leads to a form QSDE for $V$, however the Banach-Steinhaus Theorem does not help in this case. The above result therefore also fills a gap in the proof of theorem 2.4 of $\left[\mathbf{F a}_{2}\right]$.

From this result and part (c) of Theorem 2.3 we may now give necessary and sufficient conditions for a contraction cocycle to satisfy a QSDE.

THEOREM 4.2. Let $V$ be a strongly continuous left contraction cocycle on $\mathfrak{h}$ with noise dimension space $\mathrm{k}$. Then the following are equivalent:

(i) $\mathfrak{D}^{V, T}$ is dense in $\mathfrak{h}$ for some total subset $\mathrm{T}$ of $\mathrm{k}$ containing 0 ;

(ii) $V$ strongly satisfies a QSDE of the form (2.2) on some domain of the form $\mathfrak{D}_{0} \otimes$ $\varepsilon(0)+\mathfrak{D} \otimes \mathcal{E}_{\mathrm{D}}$

(iii) $V$ is a $\mathrm{T}^{\dagger}-\bar{T}$-solution of a form $\mathrm{QSDE}(2 \cdot 1)$ on some domain of the form $\mathfrak{D} \otimes \mathcal{E}_{\mathrm{T}}$.

Remark. Thus if $V$ is a left contraction cocycle on $\mathfrak{h}$ which satisfies a QSDE of the type (2.2) on $\mathfrak{D}^{V, 0} \otimes \varepsilon(0)+\mathfrak{D}^{V, T} \otimes \mathcal{E}_{\mathrm{D}}$, where $D=\operatorname{Lin} T$, then

$$
F=F^{V, \mathrm{D}} \text {. }
$$

\section{Coordinates}

Suppose that $\mathrm{k}$ is separable with orthonormal basis $\eta=\left(d_{i}\right)_{i} \geqslant 1$, and set $d_{0}:=0$. Let $V$ be a strongly continuous left contraction cocycle on $\mathfrak{h}$ and suppose that $\mathfrak{D}=\bigcap_{\alpha, \beta}$ Dom $G_{(\alpha, \beta)}$ is dense in $\mathfrak{h}$, where $G_{(\alpha, \beta)}$ denotes the generator $G_{c, d}^{V}$ for $c=d_{\alpha}$ and $d=d_{\beta}$. Then Theorem $4 \cdot 1$ ensures that $V$ strongly satisfies a Hudson-Parthasarathy equation

$$
d V_{t}=V_{t} F_{\beta}^{\alpha} d \Lambda_{\alpha}^{\beta}(t), \quad V_{0}=I,
$$

in which $\left[F_{\beta}^{\alpha}\right]_{\alpha, \beta \geqslant 0}$ is the matrix of components of an operator $F \in \mathcal{O}(\mathfrak{D} \otimes \widehat{D})$ where $\mathrm{D}=$ Lin $\eta$-in other words the matrix is semiregular in the sense that $\sum_{\alpha \geqslant 0}\left\|F_{\beta}^{\alpha} v\right\|^{2}<\infty$ for all $\beta \geqslant 0$ and $v \in \mathfrak{D}$. Moreover the components are recovered from the associated semigroup generators by the affine transformation

$$
\begin{aligned}
F_{0}^{0} & =G_{(0,0)} \\
F_{0}^{i} & =G_{(i, 0)}-G_{(0,0)}+\frac{1}{2}, \quad i \geqslant 1 \\
F_{j}^{0} & =G_{(0, j)}-G_{(0,0)}+\frac{1}{2}, \quad j \geqslant 1 \\
F_{j}^{i} & =G_{(i, j)}-G_{(i, 0)}-G_{(0, j)}+G_{(0,0)}-\delta_{j}^{i}, \quad i, j \geqslant 1,
\end{aligned}
$$

$\delta_{j}^{i}$ being the Kronecker delta. 
Cocycles from stochastic generators

Our treatment of the existence question for (2.2) is founded on the following infinitesimal version of Theorem 1.7.

Proposition 4.3. Let $Q_{\top}=\left\{Q^{c, d}: c, d \in \mathrm{T}\right\}$ be a family of $C_{0^{-}}$contraction semigroups on $\mathfrak{h}$, indexed by a total subset $\mathrm{T}$ of $\mathrm{k}$ containing 0 and let $G_{c, d}$ denote the generator of the semigroup $Q^{c, d}$. Suppose that there is a sequence of strongly continuous left contraction cocycles $\left(V^{(n)}\right)$ on $\mathfrak{h}$ and, for each $c, d \in \mathrm{T}$, a core $\mathfrak{D}_{c, d}$ for $G_{c, d}$ such that:

(a) $\mathfrak{D}_{c, d} \subset \mathfrak{D}^{V^{(n)}, T}$ for each $n \in \mathbb{N}$, and

(b) $E^{\widehat{c}} F^{(n)} E_{\widehat{d}}-\chi(c, d) \rightarrow G_{c, d}$ pointwise on $\mathfrak{D}_{c, d}$, for all $c, d \in \mathrm{T}$, where $F^{(n)}:=F^{\left.V^{(n)}\right)}, \mathrm{T}$. Then there is a unique strongly continuous left contraction cocycle $V$ whose associated semigroups include $Q_{\mathrm{T}}$. Moreover $V^{(n)} \rightarrow V$ in the weak operator topology.

Proof. We use the notation ${ }^{(n)} Q^{c, d}$ and $G_{c, d}^{(n)}$ for semigroups and generators associated with the cocycle $V^{(n)}$. Condition (a) and Corollary 1.4 imply that $\mathfrak{D}_{c, d} \underline{\otimes} \widehat{\mathrm{D}} \subset$ Dom $F^{(n)}$, where $\mathrm{D}=\mathrm{Lin} \mathrm{T}$, so each $F^{(n)}$ is densely defined, and also $E^{\widehat{c}} F^{(n)} E_{\widehat{d}}-\chi(c, d) \subset G_{c, d}^{(n)}$ by (1.20). Hence, by the Trotter-Kato Theorem ([Dav, corollary 3.18]), assumption (b) implies that

$$
\lim _{n \rightarrow \infty} \sup _{t \in[0, T]}\left\|\left({ }^{(n)} Q_{t}^{c, d}-Q_{t}^{c, d}\right) u\right\| \longrightarrow 0
$$

for all $c, d \in \mathrm{T}, u \in \mathfrak{h}$ and $T>0$. The result therefore follows by Theorem 1.7 and Lemma $1 \cdot 2$.

Remark. This result is a stochastic generalisation of the Trotter-Kato Theorem. In the usual version pointwise convergence of the generators implies convergence of the sequence of semigroups in the strong operator topology. However a similar strengthening of the conclusion for cocycles is not possible - as can be demonstrated using the conditions for isometricity of cocycles given in terms of conservativity of an associated quantum dynamical semigroup. See $[\mathbf{L W}]$ for details.

THEOREM 4.4. Let $F \in \mathcal{O}(\mathfrak{D} \underline{\otimes} \widehat{\mathrm{D}})$ where $\mathfrak{D}$ is a dense subspace of $\mathfrak{h}$ and $\mathrm{D}=$ Lin $\mathrm{T}$ for a total subset $\mathrm{T}$ of $\mathrm{k}$ containing 0 . Assume that:

(a) for each $c, d \in \mathrm{T}, E^{\widehat{c}} F E_{\widehat{d}}-\chi(c, d)$ is a pregenerator of a $C_{0}$-contraction semigroup $Q^{c, d} ;$ and

(b) there is a sequence $\left(F^{(n)}\right)$ in $B(\mathfrak{h} \otimes \widehat{\mathrm{k}})$ satisfying the operator inequality (2.5), such that, for all $c, d \in \mathrm{T}$,

$$
E^{\widehat{c}} F^{(n)} E_{\widehat{d}} \longrightarrow E^{\widehat{c}} F E_{\widehat{d}} \text { pointwise on } \mathfrak{D} \text {. }
$$

Then $F \subset F^{V, T}$ for a unique strongly continuous left contraction cocycle $V$ on $\mathfrak{h}$. Moreover, for all $c \in \mathrm{k}$ and $d \in \mathrm{T}$,

$$
\begin{aligned}
& G_{c, d}^{V}=\overline{E^{\widehat{c}} F E_{\widehat{d}}-\chi(c, d)}, \text { and } \\
& G_{d, c}^{\widetilde{V}} \supset E^{\widehat{d}} F^{*} E_{\widehat{c}}-\chi(d, c) .
\end{aligned}
$$

Proof. By Theorems $2 \cdot 3$ and $4 \cdot 1$, assumption (a) (with $c=d=0$ ) implies uniqueness. Let $G_{c, d}$ be the generator of $Q^{c, d}$ and let $V^{(n)}$ be the strongly continuous left contraction cocycle generated by $F^{(n)}$ (see the remark following Proposition 2.1). Then the hypotheses of Proposition 4.3 are satisfied with $\mathfrak{D}_{c, d}=\mathfrak{D}$ for each $c, d \in \mathrm{T}$. Let $V$ be the resulting cocycle. Then $G_{c, d}^{V}=G_{c, d}$ so $E^{\widehat{c}} F E_{\widehat{d}} \subset G_{c, d}^{V}+\chi(c, d)$ and therefore $\mathfrak{D}^{V, \boldsymbol{T}} \supset \mathfrak{D}$. This gives 
$F^{V, \mathrm{~T}} \supset F$ and so $G_{c, d}^{V} \supset \overline{E^{\widehat{c}} F E_{\widehat{d}}-\chi(c, d)}$ for all $c \in \mathrm{k}$ and $d \in \mathrm{D}$, by (1.20). Now $\mathfrak{D}$ is a core for $G_{c, d}^{V}$ when $c, d \in \mathrm{T}$ so, by part (b) of Proposition $1 \cdot 3$, it is also a core when $c \in \mathrm{k}$. The above inclusion is therefore an equality. It remains only to verify the inclusion $(4 \cdot 2)$, but since $G_{d, c}^{\widetilde{V}}=\left(G_{c, d}^{V}\right)^{*}$ this follows by taking adjoints.

Remarks. Under the conditions of the theorem, if also Dom $F^{*} \supset \mathfrak{D}^{\dagger} \otimes \widehat{\mathrm{D}^{\dagger}}$, for dense subspaces $\mathfrak{D}^{\dagger}$ and $\mathrm{D}^{\dagger}$ of $\mathfrak{h}$ and $\mathrm{k}$ respectively, then $\mathfrak{D}^{\widetilde{V}, \mathrm{D}^{\dagger}}$ contains the dense subspace $\mathfrak{D}^{\dagger}$ so $\widetilde{V}$ strongly satisfies the QSDE (2.2) with coefficient $F^{\widetilde{V}, D^{\dagger}}$ on $\mathfrak{D}^{\widetilde{V}, 0} \otimes \varepsilon(0)+\mathfrak{D}^{V, \mathrm{D}^{\dagger}} \otimes \mathcal{E}_{\mathrm{D}^{\dagger}}$, and $F^{*} \supset F^{\widetilde{V}, \mathrm{D}^{\dagger}}$. In particular, $F^{*}$ satisfies the form inequality (2.4) on $\mathfrak{D}^{\widetilde{V}, 0} \oplus\left(\mathfrak{D}^{\widetilde{V}, \mathrm{D}^{\dagger}} \underline{\otimes} \mathrm{D}^{\dagger}\right)$.

Our next result extends Fagnola's existence theorem ([Fa $]$ ). Whereas his proof requires separability of both of the Hilbert spaces $\mathfrak{h}$ and $k$, ours requires a strengthening of his condition which amounts to $K$ being a pregenerator of a $C_{0}$-semigroup. This difference in hypotheses reflects our difference of approach. Whereas he approximates the solution process by adapting Frigerio's diagonalisation argument with the Arzelà-Ascoli Theorem to cocycles constructed from bounded stochastic generators, we approximate a sufficient number of the associated semigroup generators by exploiting the Trotter-Kato Theorem and this demands stronger core requirements.

THEOREM 4.5. Let $F \in \mathcal{O}(\mathfrak{D} \otimes \underline{\mathrm{D}})$, with block matrix form $\left[\begin{array}{cc}K & M \\ L & C-I\end{array}\right]$, where $\mathfrak{D}$ is a dense subspace of $\mathfrak{h}$ and $\mathrm{D}=\mathrm{Lin} \mathrm{T}$ for a total subset $\mathrm{T}$ of $\mathrm{k}$ containing 0 . Suppose that:

(a) $2 \operatorname{Re}\langle\xi, F \xi\rangle+\|\Delta F \xi\|^{2} \leqslant 0$ for all $\xi \in \mathfrak{D} \underline{\otimes} \widehat{\mathrm{D}} ;$ and

(b) $K+M E_{d}-\frac{1}{2}\|d\|^{2}$ is a pregenerator of a $\bar{C}_{0}$-semigroup, for each $d \in \mathrm{T}$.

Then $F \subset F^{V, T}$ for a unique strongly continuous left contraction cocycle $V$ on $\mathfrak{h}$, moreover (4.1) and (4.2) hold.

Proof. By Proposition 3.1, assumption (a) implies that $G_{c, d}^{0}:=E^{\widehat{c}} F E_{\widehat{d}}-\chi(c, d)$ is dissipative and $G_{c, d}:=\overline{G_{c, d}^{0}}$ generates a $C_{0}$-contraction semigroup if and only if $G_{b, d}$ does, for $b, c \in \mathrm{k}$ and $d \in \mathrm{D}$. Since $K+M E_{d}-1 / 2\|d\|^{2}=G_{0, d}^{0}$, this operator is dissipative for each $d \in \mathrm{D}$ and assumption (b) is equivalent to $G_{0, d}$ being a $C_{0}$-semigroup generator for each $d \in \mathrm{T}$. Therefore $G_{c, d}$ is such a generator for each $c \in \mathrm{k}$ and $d \in \mathrm{T}$. In view of Proposition $3 \cdot 2$, Theorem $4 \cdot 4$ therefore applies.

COROLLARY 4.6. If condition (b) of Theorem 4.5 is replaced by:

(bi) $\operatorname{Ran}(\lambda I-K)$ is dense in $\mathfrak{h}$ for some $\lambda>0$; and

(bii) $M E_{d}$ is $K$-bounded, for each $d \in \mathrm{T}$,

then the conclusion of the theorem holds, moreover

$$
\text { Dom } G_{e, d}^{V} \supset \text { Dom } \bar{K} \text { for all } e \in \mathrm{k} \text { and } d \in \mathrm{D} \text {, }
$$

with equality when $d$ is a sufficiently small multiple of an element of $\mathrm{T}$.

Proof. Since $K$ is dissipative (bi) is equivalent to $\bar{K}$ being a $C_{0}$-contraction semigroup generator, by the Lumer-Phillips Theorem ([Dav, theorem 2.25]). For $\alpha>0$ let $\mathrm{T}^{\alpha}=\{(\alpha+$ $\left.\left.\lambda_{d}\right)^{-1} d: d \in \mathrm{T}\right\}$, where $\lambda_{d}$ is the relative bound of $M E_{d}$ with respect to $K$, thus Lin $\mathrm{T}^{\alpha}=\mathrm{D}$ and $M E_{d}$ has relative bound less than one for each $d \in \mathrm{T}^{\alpha}$. Using Proposition $3 \cdot 1$ once more this means that, for each $e \in \mathrm{k}$ and $d \in \mathrm{T}^{\alpha}, E^{\widehat{e}} F E_{\widehat{d}}-\chi(e, d)$ is a relatively bounded perturbation of $K$ with relative bound less than one. Therefore, by Gustafson's Theorem, its closure has the same domain as $\bar{K}$ and is a $C_{0}$-contraction semigroup generator, so (b) of 
Theorem 4.5 holds (with $\mathrm{T}^{\alpha}$ in place of $\mathrm{T}$ ) and the theorem applies. We have

$$
\text { Dom } G_{e, d}^{V}=\operatorname{Dom} \bar{K} \text { for } e \in \mathrm{k}, d \in \mathrm{T}^{\alpha} \text { and } \alpha>0 \text {. }
$$

The proof therefore follows by Corollary 1.4 since $\mathrm{D}=\operatorname{Lin} T^{\alpha}$.

Remark. By choosing suitable functions $\mu$ and $\lambda$ in the example below, it is possible to find an operator $F \in \mathcal{O}(\mathfrak{D} \otimes \widehat{D})$ satisfying the conditions of Theorem 4.5 but whose coefficients $M_{d}$ are not $K$-bounded, so that $F$ is not covered by the corollary above.

Dualising we obtain alternative conditions.

COROLlary 4.7. Let $F \in \mathcal{O}(\mathfrak{D} \otimes \widehat{\mathrm{D}})$ and $F^{\dagger} \in \mathcal{O}\left(\mathfrak{D}^{\dagger} \otimes \widehat{\mathrm{D}^{\dagger}}\right)$ be densely defined operators on $\mathfrak{h} \otimes \widehat{\mathrm{k}}$ with block matrix forms $\left[\begin{array}{cc}K & M \\ L & C-I\end{array}\right]$ and $\left[\begin{array}{cc}K^{\dagger} & L^{\dagger} \\ M^{\dagger} & C^{\dagger}-I\end{array}\right]$ respectively, satisfying $F^{*} \supset F^{\dagger}$, where $\mathrm{D}=\mathrm{Lin} \mathrm{T}$ for a total subset $\mathrm{T}$ of $\mathrm{k}$ containing 0 . Then the conclusions of Theorem 4.5 hold under the conditions:

(a) $F$ and $F^{\dagger}$ satisfy the form inequality (2.4);

(bi) $K^{\dagger}$ is a pregenerator of a $C_{0}$-semigroup on $\mathfrak{h}$; and

(bii) $\mathfrak{D}$ is a core for the operator $\left(K^{\dagger}+E^{d} M^{\dagger}-(1 / 2)\|d\|\right)^{*}$, for each $d \in \mathrm{T}$.

Proof. In view of assumption (bi), Proposition 3.1 applied to $F^{\dagger}$ shows that $\left(K^{\dagger}+E^{d} M^{\dagger}-\right.$ $\left.(1 / 2)\|d\|^{2}\right)$ is a pregenerator of a $C_{0}$-contraction semigroup. Assumption (bii) therefore implies that its closure is $\left(K+M E_{d}-(1 / 2)\|d\|^{2}\right)^{*}$, thus $\left(K+M E_{d}-(1 / 2)\|d\|^{2}\right)$ is a pregenerator of a $C_{0}$-contraction semigroup and so Theorem 4.5 applies.

Many examples are covered by the following consequence of Theorem 4.5, with $C$ typically being unitary.

THEOREM 4.8. Let $H$ be a closed symmetric operator on $\mathfrak{h}, L$ a closed operator $\mathfrak{h} \rightarrow$ $\mathfrak{h} \otimes \mathrm{k}, C$ a contraction operator on $\mathfrak{h} \otimes \mathrm{k}$ and $\mathrm{T}$ a total subset of $\mathrm{k}$ containing 0 , such that $\mathfrak{D}:=$ Dom $H \cap$ Dom $L^{*} L \cap \bigcap_{d \in \mathrm{T}}$ Dom $L^{*} C E_{d}$ is dense in $\mathfrak{h}$, and let $F=\left.\left[\begin{array}{cc}K & -L^{*} C \\ L & C-I\end{array}\right]\right|_{\mathfrak{D} \otimes} \widehat{\mathrm{D}}$ where $K=i H-(1 / 2) L^{*} L$ and $\mathrm{D}=\mathrm{Lin} \mathrm{T}$. Then the following hold.

(a) (i) F satisfies (2.4), with equality if and only if $C$ is isometric.

(ii) If there are constants $\gamma_{d}>0(d \in \mathrm{T})$ such that

$$
\left(\gamma_{d} I+\frac{1}{2} L^{*} L+L^{*} C E_{d}-i H\right) \mathfrak{D} \text { is dense in } \mathfrak{h} .
$$

then $F$ generates a strongly continuous left contraction cocycle on $\mathfrak{h}$.

(b) Suppose that $\mathfrak{D}^{\dagger}:=$ Dom $H^{*} \cap$ Dom $L^{*} L \cap \bigcap_{d \in T^{\dagger}}$ Dom $L^{*} E_{d}$ is dense in $\mathfrak{h}$, and let $\mathrm{D}^{\dagger}=$ Lin $\mathrm{T}^{\dagger}$ for another total subset of $\mathrm{k}$ containing 0 . Then:

(i) $F^{*}$ satisfies $(2.4)$ on $\mathfrak{D}^{\dagger} \underline{\otimes} \widehat{\mathrm{D}^{\dagger}}$, with equality if and only if $C$ is coisometric on $\mathfrak{h} \otimes \mathrm{k}$

(ii) if there are constants $\gamma_{d}>0\left(d \in \mathrm{T}^{\dagger}\right)$ such that

$$
\left(\gamma_{d} I+\frac{1}{2} L^{*} L-L^{*} E_{d}+i H^{*}\right) \mathfrak{D}^{\dagger} \text { is dense in } \mathfrak{h}
$$

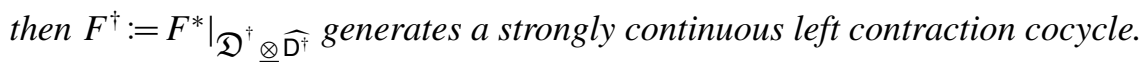

Examples in which $C=I$ and $H=0$ have arisen recently in the problem of constructing stochastic dilations of tracially-symmetric quantum Markov semigroups ([GS $\mathbf{G}])$. In this case it suffices for $\mathfrak{D}$ to be a core for the positive selfadjoint operator $L^{*} L$, and for $L^{*} E_{d}(d \in \mathrm{T})$ to be relatively bounded with respect to $L^{*} L$. 
Let $\mathfrak{D}$ be the linear span of the standard orthonormal basis of $\mathfrak{h}:=l^{2}\left(\mathbb{Z}_{+}\right)$, let

$$
F=\left[\begin{array}{cc}
\nu(N) & W^{*} \bar{\lambda}(N) \\
-\lambda(N) W & 0
\end{array}\right], \text { where } v(n)=i \mu(n)-\frac{1}{2}|\lambda|^{2}(n+1),
$$

where $W$ and $N$ denote respectively the isometric right shift on $\mathfrak{h}$ and the number operator on $\mathfrak{h}$, and $\lambda: \mathbb{Z}_{+} \rightarrow \mathbb{C}$ and $\mu: \mathbb{Z}_{+} \rightarrow \mathbb{R}$ are arbitrary functions. Then $F^{*}+F+F^{*} \Delta F$ and $F+F^{*}+F \Delta F^{*}$ both vanish on $\mathfrak{D} \oplus \mathfrak{D}$ and Theorem 4.5 applies. Models of this type arise in the study of inverse harmonic oscillators interacting with a heat bath in the singular coupling limit ([Wal]). Conditions on the pair $(\lambda, \mu)$ which ensure isometry/unitarity of the resulting contraction cocycle are investigated in $[\mathbf{F a W}]$, from the point of view of the right equation $d U_{t}=\left(F^{*} \otimes I_{\mathcal{F}}\right) \widehat{U}_{t} d \Lambda_{t}$.

Classical birth and death processes have been constructed using quantum stochastic calculus $\left(\left[\mathbf{F a}_{1}, \mathbf{F a W}\right]\right)$. These are similarly covered by the above theorem, this time working with the Hilbert space $l^{2}(\mathbb{Z})$ and two dimensional quantum noise. These examples and others are treated in detail in $\left[\mathbf{L W}_{\mathbf{4}}\right]$.

Acknowledgements. We are grateful to Nick Weatherall for drawing our attention to Dixmier's counterexamples. SJW acknowledeges financial support for this work from two EU TMR Networks (HPRN-CT-2002-00279 and HPRN-CT-2002-00280).

\section{REFERENCES}

[AJL] L. ACCARdi, J.-L. Journé and J. M. Lindsay. On multidimensional Markovian cocycles. In Quantum Probability and Applications IV, eds. L. Accardi \& W. von Waldenfels. Lecture Notes in Math. 1396 (Springer, 1989), pp. 59-67.

[Bou] N. BOURBAKI. Elements of Mathematics - Topological Vector Spaces (Springer-Verlag, 1987).

[Dav] E. B. DAVIES. One-parameter Semigroups (Academic Press, 1980).

[EfR] E. G. EFFros and Z.-J. RUAN. Operator Spaces (Oxford University Press, 2000).

[Fa $]$ F. Fagnola. Pure birth and death processes as quantum flows in Fock space. Sankhyā Ser. A $\mathbf{5 3}$ (1991), 288-297.

[Fa2] F. FAGNOLA. Characterization of isometric and unitary weakly differentiable cocycles in Fock space. In Quantum Probability and Related Topics VIII, ed. L. Accardi. (World Scientific, 1993), pp. 143-164.

[FaW] F. FAGNOLA and S. J. WiLLS. Solving quantum stochastic differential equations with unbounded coefficients. J. Funct. Anal. 198 (2003), 279-310.

[GS 1 D. Goswami and K. B. SINHA. Hilbert modules and stochastic dilation of a quantum dynamical semigroup on a von Neumann algebra. Comm. Math. Phys. 205 (1999), 377-405.

[GS $\left.\mathbf{G}_{2}\right]$ D. GoswamI and K. B. SinHA. Stochastic dilation of symmetric completely positive semigroups arXiv:math-ph/0201005. (See also Quantum Stochastic Processes and Noncommutative Geometry, Cambridge Tracts in Mathematics 169 (Cambridge University Press, 2007).)

[HuL] R. L. HUDSON and J. M. LINDSAY. On characterizing quantum stochastic evolutions. Math. Proc. Camb. Phil. Soc. 102 (1987), 363-369.

[HuP] R. L. HUDSON and K. R. PARThASARATHY. Quantum Itô's formula and stochastic evolutions. Comm. Math. Phys. 93 (1984), 301-323.

[Jou] J.-L. Journé. Structure des cocycles markoviens sur l'espace de Fock. Probab. Theory Related Fields 75 (1987), 291-316.

[Lie] V. Liebscher. How to generate Markovian cocycles on boson Fock space. Infin. Dimens. Anal. Quantum Probab. Relat. Top. 4 (2001), 215-219.

[L] J. M. LINDSAY. Quantum stochastic analysis - an introduction. In Quantum Independent Increment Processes I: From Classical Probability to Quantum Stochastic Calculus, eds. U. Franz \& M. Schürmann. Lecture Notes in Math. 1865 (Springer, 2005), pp. 181-271.

$\left[\mathbf{L W}_{\mathbf{1}}\right]$ J. M. LINDSAY and S. J. WILLS. Existence, positivity, and contractivity for quantum stochastic flows with infinite dimensional noise. Probab. Theory Related Fields 116 (2000), 505-543.

$\left[\mathbf{L W}_{2}\right]$ J. M. LINDSAY and S. J. WILLS. Markovian cocycles on operator algebras, adapted to a Fock filtration. J. Funct. Anal. 178 (2000), 269-305.

$\left[\mathbf{L W}_{3}\right]$ J. M. LINDSAY and S. J. WILLS. Quantum stochastic cocycles and completely bounded semigroups on operator spaces I, in preparation. 
$\left[\mathbf{L W}_{4}\right]$ J. M. LINDSAY and S. J. WILLS. Construction of some quantum stochastic operator cocycles by the semigroup method. Proc. Indian Acad. Sci. Math. Sci. 116 (2006), 519-529. arXiv:math.FA/0606545.

[Mey] P.-A. MeYer. Quantum Probability for Probabilists. 2nd Edition. Lecture Notes in Math. 1538 (Springer, 1993).

[Moh] A. MOHARI. Quantum stochastic differential equations with unbounded coefficients and dilations of Feller's minimal solution. Sankhyā Ser. A $\mathbf{5 3}$ (1991), 255-287.

[MoP] A. Mohari and K. R. PARThASARATHY. A quantum probabilistic analogue of Feller's condition for the existence of unitary Markovian cocycles in Fock spaces. In Statistics and Probability: A Raghu Raj Bahadur Festschrift, eds. J. K. Ghosh, S. K. Mitra, K. R. Parthasarathy \& B. L. S. Prakasa Rao. (Wiley Eastern, 1993), pp. 475-497.

[Pis] G. PISIER. Introduction to Operator Space Theory. London Mathematical Society Lecture Note Series 294 (Cambridge University Press, 2003).

[ReS] M. ReED and B. Simon. Methods of Modern Mathematical Physics II: Fourier Analysis, Selfadjointness (Academic Press, 1975).

[Wal] W. vON WALDENFELs. Continuous Maassen kernels and the inverse oscillator. In Séminaire de Probabilités XXX, eds. J. Azéma, M. Emery \& M. Yor. Lecture Notes in Math. 1626 (Springer, 1996), pp. 117-161. 